\title{
3D Printed Bionic Nanodevices
}

Yong Lin Konga*, Maneesh K. Gupta ${ }^{\mathrm{b}}$, Blake N. Johnson ${ }^{\mathrm{c}}$, Michael C. McAlpine ${ }^{\mathrm{d} *}$

${ }^{a}$ Koch Institute for Integrative Cancer Research, Massachusetts Institute of Technology, Cambridge, Massachusetts 02139, USA

${ }^{b}$ Air Force Research Laboratories, Wright-Patterson Air Force Base, Ohio 45433, USA

${ }^{c}$ Department of Industrial and Systems Engineering, Virginia Tech, Blacksburg, Virginia 24061, USA

${ }^{d}$ Department of Mechanical Engineering, University of Minnesota, Minneapolis, Minnesota 55455, USA

*Corresponding authors. Tel.: Kong: (609) 356-3699 / McAlpine: (609) 542-0275

E-mail: yongkong@mit.edu / mcalpine@umn.edu

KEYWORDS: 3D printing, 4D printing, bionic devices, nanomaterials, nanodevices, bioelectronics, cyborgs, bio-nano hybrids, electronic skins, active functional devices 


\section{Summary}

The ability to three-dimensionally interweave biological and functional materials could enable the creation of bionic devices possessing unique and compelling geometries, properties, and functionalities. Indeed, interfacing high performance active devices with biology could impact a variety of fields, including regenerative bioelectronic medicines, smart prosthetics, medical robotics, and human-machine interfaces. Biology, from the molecular scale of DNA and proteins, to the macroscopic scale of tissues and organs, is three-dimensional, often soft and stretchable, and temperature sensitive. This renders most biological platforms incompatible with the fabrication and materials processing methods that have been developed and optimized for functional electronics, which are typically planar, rigid and brittle. A number of strategies have been developed to overcome these dichotomies. One particularly novel approach is the use of extrusion based multi-material 3D printing, which is an additive manufacturing technology that offers a freeform fabrication strategy. This approach addresses the dichotomies presented above by (1) using 3D printing and imaging for customized, hierarchical, and interwoven device architectures; (2) employing nanotechnology as an enabling route for introducing high performance materials, with the potential for exhibiting properties not found in the bulk; and (3) 3D printing a range of soft and nanoscale materials to enable the integration of a diverse palette of high quality functional nanomaterials with biology. Further, 3D printing is a multi-scale platform, allowing for the incorporation of functional nanoscale inks, the printing of microscale features, and ultimately the creation of macroscale devices. This blending of 3D printing, novel nanomaterial properties, and 'living' platforms may enable next-generation bionic systems. In this review, we highlight this synergistic integration of the unique properties of nanomaterials with the versatility of extrusionbased 3D printing technologies to interweave nanomaterials and fabricate novel bionic devices. 


\section{Introduction}

The synergistic integration of biological systems with electronic materials and devices could enable the creation of novel bionic devices. Due to the increasing miniaturization and proliferation of portable electronic devices, the field of bionics has transitioned from science fiction to an area of increasing scientific interest, with particular relevance to the fields of regenerative medicines, smart prosthetics, medical robotics and human-machine interfaces [1-4]. Most research in the field of bionics to date has focused on developing robots which behave increasingly more like humans. Similarly, an equally compelling challenge is integrating electronic and robotic components in a seamless manner onto the human body. For example, bioelectronic medicines and devices could potentially be utilized to restore or even augment the complex functionalities of naturally evolved biological systems. At the fundamental level, there are inherent materials compatibility challenges associated with integrating functional electronic materials with biology.

The term "bionics" is defined by Dictionary.com as, "utilizing electronic devices and mechanical parts to assist humans in performing difficult, dangerous, or intricate tasks, by supplementing or duplicating parts of the body [5]." Broadly speaking, "bionics" encompasses the functionalities of classes of systems that are formed by merging biological systems, which could be single cellular or multi-cellular systems [2,6-8], with engineered functional electronic and/or mechanical systems [2]. Our ability to develop tools which overcome the limitations of human biology has played a key role in survival and evolution [9]. Utilizing devices for regenerative medicine and as prosthetics can be traced back millennia [1]. Indeed, a very primitive bionic device from the first century AD involved the use of wrought iron for dental replacements [10]. Subsequently, bionic devices such as iron prosthetic hands (1504), contact lenses (1888), and artificial hip replacements (1905) have been used to restore or augment human function [1]. Over the past several decades, 
the development of active microelectronic devices has enabled the incorporation of sensing modalities [11,12], optoelectronics [13,14], actuation [15] and computational devices [16] into previously passive mechanical constructs. This has enabled an extension of the role of bionic devices toward mimicking or even augmenting the complex functionalities of biological organs. These powerful developments have been leveraged to fabricate active bionic devices such as the cochlear implant $[17,18]$ to restore hearing (Figure 1A), pacemakers and heart replacements [1] to sustain blood flow (Figure 1B), locally powered prosthetic devices [19] to provide mobility to amputees (Figure 1C), retinal implants to provide partial restoration of vision loss due to diseases such as retinitis pigmentosa [20,21] (Figure 1D), dura mater for the spinal cord [22] (Figure 1E), and digital skin sensors and electronic skins [12,23-25] (Figure 1F). Indeed, the ability to merge a diverse palette of materials classes could enable the generation of functional devices that mimic the complex functionalities of grown biological organs [15].

An optimized bionic device should be seamlessly merged with the human body in order to restore or augment human capabilities without causing side effects such as discomfort, infection [26] or rejection due to foreign body responses by the host [27-29]. While the continual discovery of new materials and novel properties will eventually lead to more optimized devices, ideality has been punctuated by challenges in integrating high performance materials and devices with biology. Three key challenges can be identified. First, the mechanical properties of high quality electronic materials are typically disparate from biology. For example, the typical Young's modulus of inorganic electronics is on the order of 1-100 GPa ( $\mathrm{Si} \sim 170 \mathrm{GPa}$ ) [30]. By contrast, the Young's modulus of skin is on the order of 0.1-1 MPa [31]. Similarly, inorganic electronic materials typically fracture at strains (ca. 1\%) [32] of up to 30× lower than human skin [33]. These significant 
differences in mechanical properties not only lead to obstacles in the integration of bionic devices onto the body, but can cause discomfort, agitation, rejection and injuries [34].

Second, the processing conditions inherent to high performance electronics are often incompatible with biology. Microelectronics are typically fabricated via "top down" approaches which can involve harsh chemical and temperature processing conditions. In contrast, organs and tissues have been grown from the "bottom up" under finely tuned physiological conditions [35]. Third and finally, electronic wafers are two-dimensional planar structures, whereas biology possesses intricately complex three-dimensional geometries from the molecular scale to the macroscale. These incompatibilities collectively present significant barriers in grafting independently fabricated bionic devices onto biology in a seamless manner.

A variety of novel strategies have been developed to address these issues, such as integration via intelligent device design $[11,32,36,37]$, transfer printing processes [13,38-41] and/or assembly of prefabricated devices [42] onto three-dimensional constructs to accommodate the geometrical and material incompatibilities. This review highlights a relatively new concept in achieving a synergistic integration of bionic devices with biology: by using 3D printing. Extrusion-based 3D printing technologies may overcome the three specific challenges mentioned above. First, the use of nanoscale materials as inks in the 3D printing process and the co-printing of soft materials and functional nanoscale inks allows for a route which minimizes mechanical discrepancies. Second, while the materials may be synthesized and/or processed under harsh, high temperature conditions in order to create high quality functional nanomaterials, the printing process is typically performed under ambient conditions via a bottom-up assembly process. Finally, the 3D printing process naturally allows for the hierarchical assembly of functional materials in three dimensions, 
commensurate with biology. An additional benefit of 3D printing is the ability to achieve a multiscale manufacturing approach built into the process.

This review article will first describe this multi-scale fabrication approach and highlight the unique properties of nanoscale conductive, semiconducting and plasmonic materials. Next, we will describe the challenges and strategies associated with the microscale printing and assembly of these nanoscale functional materials. We will then review progress to date in the use of 3D printing to create unique bionic architectures at various length scales. 


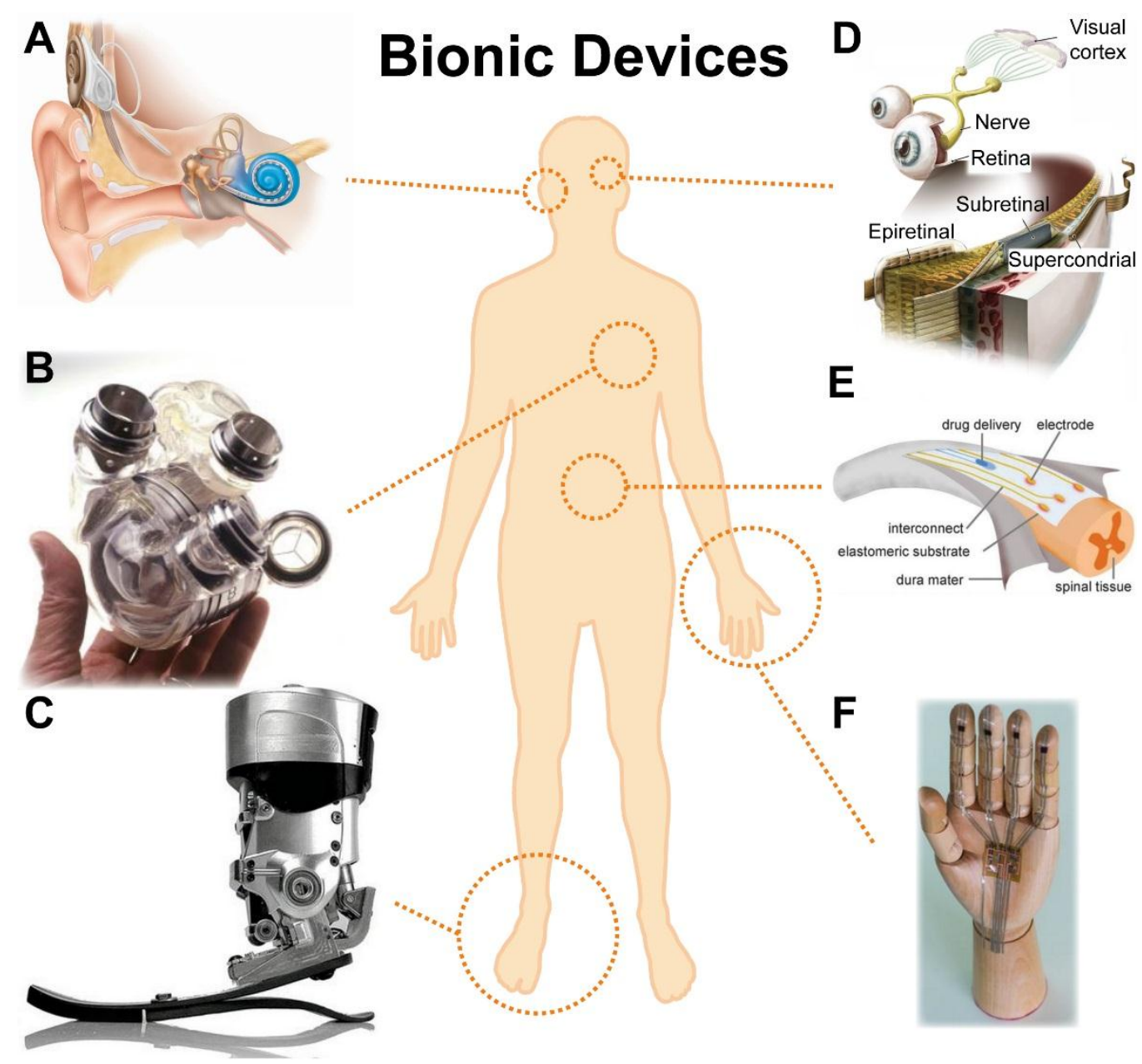

Figure 1. Bionic technologies for restorative medicine. (A) Cochlear implant [18]. (B) AbioCor self-contained replacement heart [1]. (C) Powered ankle-foot prosthetic controlled by a neuromuscular model [19]. (D) Epiretinal, subretinal, and suprachoroidal implants [21]. (E) Electronic dura mater, "e-dura," tailored for the spinal cord [22]. (F) A skin-inspired digital mechanoreceptor, where the image shows a model hand with DiTact sensors on the fingertips connected with stretchable interconnects [12]. Reprinted with permission from Refs. [18], [1], [19], [21], [22], [12], respectively. Copyright 2009 Nature Publishing Group, 2002 American Association for the Advancement of Science, 2010 IEEE, 2013 American Association for the Advancement of Science, 2015 American Association for the Advancement of Science. 


\section{D Printing for Multiscale Manufacturing}

3D printing is an additive manufacturing process used to build three-dimensional structures from computer-aided design (CAD) models in a layer-by-layer fashion. Developed since the 1980s [43], $3 \mathrm{D}$ printing has the capability to create unique architectures that cannot be made with conventional molding or subtractive manufacturing techniques. Further, the co-development of 3D imaging technologies, such as 3D scanning, allows for the acquisition of three-dimensional topological data that precisely reproduces a three-dimensional object and the incorporation of three-dimensional templates for the conformal printing of devices on non-planar substrates [44-46].

3D printing is commonly associated with either light-based or ink-based printing techniques [47]. Light-based techniques are founded on processes such as UV curing $[43,48,49]$ and two-photon polymerization [50]. Ink-based printing can be achieved via extrusion printing, inkjet printing [51], and electro-hydrodynamic printing [52]. Extrusion-based 3D printing is a particularly interesting subset of additive manufacturing in which the materials are extruded through a nozzle [47,53-57]. Such a platform is highly versatile, affordable [58] and can be readily expanded to incorporate multiple materials [59-61]. Moreover, in contrast to other printing methods, such as inkjet printing (where the typical viscosity is limited to ca. $2-10^{2} \mathrm{mPa} \cdot \mathrm{s}$ [51]), extrusion-based 3D printing is capable of incorporating a wide range of materials with viscosities up to $10^{6} \mathrm{mPa} \cdot \mathrm{s}$ and with disparate properties [47,61]. This versatility has enabled the accommodation of different classes of materials encompassing a wide range of length-scales: including nanomaterials [61,62], fibers [63], cells [64,65], tissues [66], organs [55,67], ceramics [68,69], metals [70] and polymers such as elastomers [59,60,71], gels [72,73], and biomaterials [55,74].

Nanomaterials represent novel building blocks in the toolset of 3D printed functionalities. These are materials which are confined such that at least one of their length scales is in the range of ca. 
1-100 nm. As conceived by Richard Feynman in 1959 [75], assembling materials from the bottomup has become an important assembly strategy for nanoscale materials [76], enabled by the ability to make such materials using scalable synthetic methods [77-81]. Functional nanomaterials can be dispersed into solvents to form solution-processable inks, as shown in Figure 2A, which can be extruded from nozzles to create microscale features (Figure 2B) commensurate with typical biological length scales. Finally, the co-printing of nanomaterials with soft, structural, and/or biological constructs enables the freeform fabrication of three-dimensional, macroscale, multimaterial functional devices as illustrated in Figure 2C. Extrusion-based 3D printing thus provides a promising platform for the interweaving of different materials and functionalities.

\section{Functional Nanoscale Inks}

Nanoscale inks are attractive for a number of reasons. Nanomaterials approach length scales such that external forces are negligible in comparison with van der Waals interactions [82]. Hence, nanomaterials can be assembled [83] or coated on arbitrarily-shaped three dimensional substrates with high adhesion. For example, a monolayer of graphene has been shown to exhibit an adhesion energy of $0.45 \mathrm{~J} \cdot \mathrm{m}^{-2}$ on a silicon oxide substrate [84]. Approaching the nanoscale, the surface-tovolume ratio increases as the particle size decreases $[85,86]$. This geometric effect has important consequences. For example, the decrease in particle size reduces the material melting temperature $[87,88]$. An example of this relationship between size and the melting point [89] is low melting point silver nanoparticles [90] that can be sintered to form a conductive path at lower temperatures than the bulk material. Nanomaterial properties are also highly tunable. In 1847, Michael Faraday discovered that the optical properties of gold colloids deviated from their bulk counterparts [91], introducing the prospect of tuning functional properties by tailoring the size of materials. The band gap of semiconducting nanomaterials is size-dependent below the Bohr exciton radius [85,86,92]. 
For example, the fluorescence of CdSe-CdS core-shell nanoparticles shifts from red to blue when the particle size decreases from $6 \mathrm{~nm}$ to $1.7 \mathrm{~nm}$ [93]. These effects offer a means of controlling the electronic and optical properties of nanomaterials by tuning their size during synthesis.

The ability to synthesize monodisperse nanowires and nanoparticles permits the development of printable inks that capture the unique properties of nanomaterials in a printable format, by suspending the nanoparticles in aqueous or organic solvents [77-81]. Stabilization in the solvent is typically achieved via the addition of polymeric materials [94] and surfactants, or via electrostatic interactions [95] to prevent aggregation and precipitation. The extrusion-based 3D printing method supports a wide range of fluid properties, and the printability can be tailored by modifying the surface tension and viscosity of the inks. Three common classes of printable inks include conducting, semiconducting and plasmonic nanomaterials. Printable conducting nanomaterials [96] can be synthesized with metallic [97] and carbon-based nanoparticles. Further, the ability to print thin conductive layers also enables the printing of transparent conductors $[98,99]$ that can function as electrodes for optoelectronic devices [100,101]. Highly conductive metals such as silver $[90,102]$ and gold $[103,104]$ are suitable printable inks which, unlike other metals such as aluminum [105], are less susceptible to oxidation. Alternatively, carbon nanomaterialbased conducting inks [98] such as graphene and carbon nanotubes have been actively explored. Graphene [106-108], an atomically thick layer of carbon atoms, is attractive due its exceptionally high intrinsic mobility $[109,110]$. A mobility as high as $5,000 \mathrm{~cm}^{2} \cdot \mathrm{V}^{-1} \cdot \mathrm{s}^{-1}$ can be achieved in printed graphene films [111]. Hybrid composites can also be formed by mixing both metal- and carbon-based inks. For instance, a highly conductive composite $\left(5.7 \times 10^{5} \Omega^{-1} \cdot \mathrm{m}^{-1}\right)$ that can be strained up to $140 \%$ has been demonstrated with a silver nanotube composite [112]. 
Printable semiconducting nanoscale inks provide a means of introducing active electronic functionality and tuning electrical and optical properties. Quantum dots (QDs) [92] are zerodimensional nanoscale crystals of semiconducting materials, in which quantum confinement often causes a deviation of properties from the bulk. For example, the emission wavelength of cadmium selenide QDs is tunable by changing the particle size [113]. QDs typically consist of an inorganic semiconductor core and a coating of ligands to confer solubility in solvents. A wider-bandgap inorganic material can also be coated as a shell to passivate the surface, thereby improving intrinsic properties such as photoluminescence quantum yield and photo-stability [78]. Further, the ability to synthesize highly monodisperse QDs with narrow size distributions leads to narrow emission spectra [114] that is useful for display devices with high color purity and saturation [115].

Plasmonic nanomaterials are metal nanoparticles that exhibit a localized surface plasmon resonance (LSPR), which is a collective oscillation of conduction band electrons in response to the electric field component of incident light. The unique optical properties such as large absorption and scattering cross-section, high sensitivity to the local dielectric environment, and enhanced electromagnetic field at the surface render plasmonic nanomaterials a highly attractive class of materials for a broad range of applications [116]. The LSPR wavelength is dependent on the composition, size, shape, coupling, and ambient dielectric of the nanoparticles. In biomedicine, plasmonic nanoparticles are used in applications for diagnostics and therapeutics. While tremendous progress has been made in the synthesis of size- and shape-controlled plasmonic nanostructures, their integration with other materials and application in solid-state devices is primarily either via direct fabrication (using various lithographic techniques) or immobilization on solid two-dimensional (2D) substrates such as silicon, glass, plastic, or paper. 


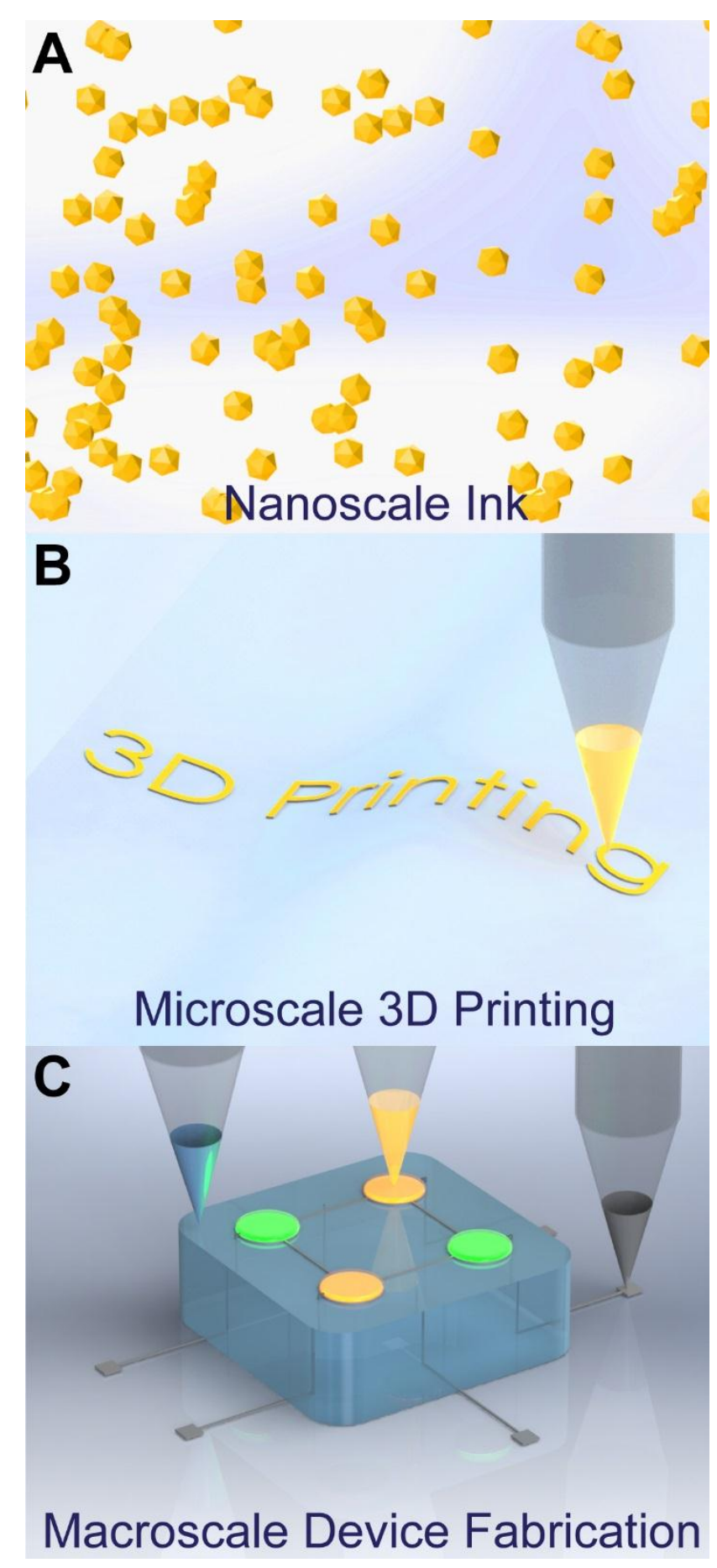

Figure 2. Multiscale, multi-material 3D printing. (A) Functional nanomaterials can be dispersed in solvents to form solution-processable inks. (B) The inks are then 3D printed at microscales via extrusion from a suitable nozzle. (C) The three-dimensional co-printing of various classes of materials enables the creation of macroscale functional devices. 


\section{Microscale 3D Printing of Inks}

The ability to pattern solution-processed nanomaterial inks can lead to the creation of devices [117119], where the properties of the film such as its thickness and morphology dictates the quality of the resulting device performance [120]. In microfabrication-based methods, this is typically accomplished via processes such as spin-coating [121], in which the liquid ink is spread uniformly via centrifugal forces. However, such processes require a rigid and flat substrate, as well as the use of photolithography for patterning. In addition, the spin coating of quantum dots expels $94-97 \%$ of the starting solution, which increases the material cost by up to 20-fold [122]. Yet, a colloidal ink printed onto a surface without spin-coating typically does not lead to a uniform film. When a droplet is left to evaporate on a substrate, the pinning of the contact line results in a non-uniform evaporation rate at the surface of the printed droplet. The enhanced drying rate at the edge of the

droplet due to the curvature difference drives the assembly of suspended particles at the edge of the evaporating solvent, resulting in so-called "coffee rings" [123,124], as shown in Figure A. The formation of multiple rings can also be observed, due to the repeated pinning and unpinning of the contact lines as the printed ink evaporates [125].

To overcome this non-homogeneous deposition, the microscale printing of nanomaterials can be achieved via directed or self-assembly based methods [83]. External forces, such as electrical forces [126], or magnetic forces [127] can be applied to drive the assembly of the particles. The assembly can also be achieved without an external field. For instance, a Marangoni effect [128130] can be introduced to drive the particle accumulation away from the edge of the droplet. This can be achieved via the addition of a co-solvent, which introduces a surface tension gradient that generates the Marangoni flow. As shown in Figure B, the introduction of $20 \%$ of dicholorobenzene to a QD ink reduces the root-mean square roughness of the resulting QD film 9-fold in comparison 
to a single-solvent ink [61]. Similarly, a surface tension gradient can be generated via the introduction of a vapor environment. For instance, the film generated from an aqueous suspension has a higher uniformity in ethanol vapor due to the strong recirculating flow generated by the surface tension gradient [131]. Alternatively, the addition of a small ionic surfactant introduces a Marangoni eddy of particles, which improves uniformity of deposition [132]. The addition of hydrosoluble polymer additives can also mitigate pinning of the contact line via an increase in viscosity and the Marangoni effect, which suppresses the coffee ring effect [133]. Coffee rings can also be reduced by introducing temperature control [134], since temperature affects the edge evaporation rate.

Interestingly, modification of the particle shape can also affect the subsequent deposition. For instance, Yunker et al. have shown that anisotropic shaped particles, such as ellipsoids, introduce strong inter-particle interactions to form loosely packed structures that prevent the accumulation of particles at the edges of the droplet [135]. Impressively, monolayer assembly of nanoparticles has been achieved by tailoring the evaporation kinetics and particle interactions at the liquid-air interface [136], as described in Figure C. For example, assembly of a large nanoparticle monolayer film $(3 \mathrm{~mm} \times 4 \mathrm{~mm})$ has been generated on a $\mathrm{Si}_{3} \mathrm{~N}_{4}$ substrate without the application of external fields. The ligands of the nanoparticles also play a critical role in this assembly approach, and excess ligand has been found to play a key role in generating the thin film using this method [136]. The coffee-ring effect can be leveraged to create high resolution patterns and features which are otherwise challenging to create via direct-writing with a 3D printer. For instance, a transparent conductive film can be formed from silver nanoparticle rings generated with the coffee ring effect. A network of silver nanoparticle rings $(10 \mu \mathrm{m}$ width, $300 \mathrm{~nm}$ height, $150 \mu \mathrm{m}$ diameter $)$ can generate a conductive film with a resistivity of $4 \times 10^{-7} \Omega \cdot \mathrm{m}$ and a transparency of $95 \%$. This 
method is not limited to silver nanoparticles, and has also been demonstrated with carbon-based particles such as carbon nanotubes [137].

Similarly, "stick-slip" motion can be used to create a uniform array of nanoparticles. Unlike the irregular ring pattern resulting from a droplet left evaporating on a surface, "bands" can be assembled by confining the evaporation rate of the inks. The exploitation of such effects have been demonstrated to yield highly regular arrays of micrometer size band structures in both planar $[138,139]$ and confined constructs $[140,141]$. Indeed, the integration of such approaches could enable the microscale patterning of nanoscale inks within a 3D printing setting. For instance, as described in Figure D, doctor blades can provide a confinement of the evaporation rate, and the manipulation of the velocity profile of the translation stage can enable the creation of highly regular microscale quantum dot stripes on a planar surface, with control over the widths and thicknesses of the quantum dot stripes [142]. 

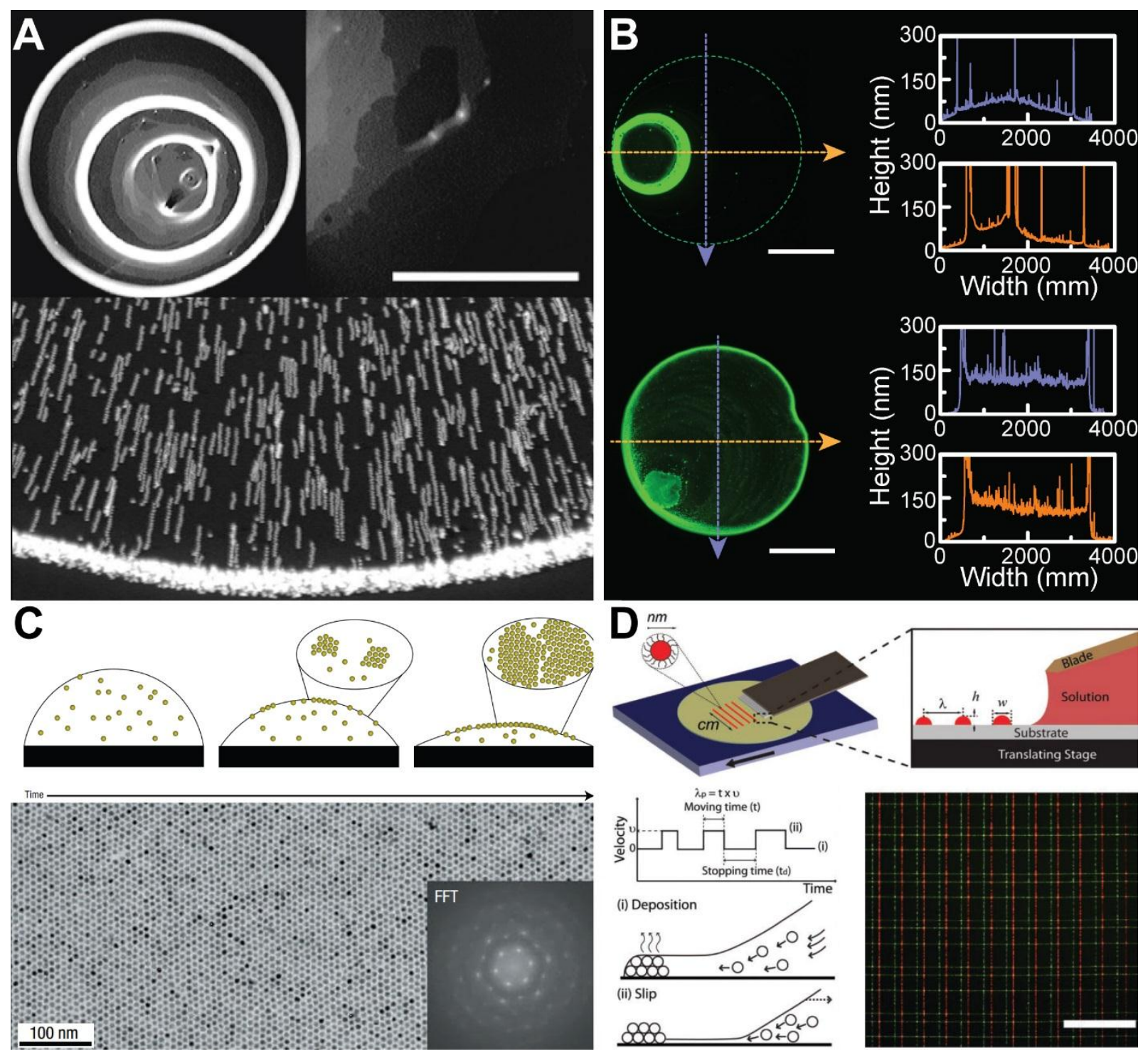

Figure 3. Microscale patterning of nanoscale inks on a surface. (A) Challenges inherent to assembling particles via convective self-assembly methods. Top left figure shows formation of socalled "coffee-rings," typically observed when a colloidal suspension droplet dries on a surface. The photograph is of a deposit left by $100 \mathrm{~nm}$ microspheres with a volume fraction of $1 \%$. Top right figure shows non-uniformity in the region of the ring, where the grey scale indicates the density of particles with the white color indicating the highest density. Scale bar is $500 \mu \mathrm{m}$ [124]. Bottom figure show the superimposed exposures that illustrate the motion of the particles toward 
the edge of the droplet during the drying process [123]. (B) Non-uniformity can be reduced by introducing a co-solvent. Top figure shows the deposition of quantum dots from pure toluene, while bottom figure shows an improvement in the morphology via the introduction of $20 \%$ dichlorobenzene [61]. Scale bar is $1 \mathrm{~mm}$. (C) Evaporation kinetics and particle interactions with the liquid-air interface can be tailored to achieve monolayer assembly of nanoparticles. Micrograph shows the monolayer produced by a solution of dodecanethiol-ligated $6 \mathrm{~nm}$ gold nanocrystals. Inset shows the fast Fourier transform (FFT) of the image [136]. (D) Arrays of quantum dots are generated via stick-slip motion of the contact line. The features are controlled by the velocity profile of the translation stage. Bottom right figure shows the fluorescent microscopy image of grid patterns of the quantum dots. Scale bar is $200 \mu \mathrm{m}$ [142]. Reprinted with permission from Refs. [124], [123], [61], [136], [142], respectively. Copyright 2000 American Physical Society, 1997 Nature Publishing Group, 2014 American Chemical Society, 2006 Nature Publishing Group, 2010 John Wiley \& Sons.

\section{Mechanical Properties of 3D Printed Architectures}

In this section, we highlight some of the unique mechanical properties enabled by extrusion-based 3D printing, which has the advantage of creating freeform architectures that can be tailored to create robust bionic devices. First, the extrusion-based 3D printing process can be combined with bioinspired inks to create lightweight composites that mimic natural materials. The co-printing of alternate layers of hard and soft materials can result in structures exhibiting outstanding strength and toughness [143] and enhanced fracture resistance [144]. Lightweight composite structures can also be achieved via shear-induced alignment of fibers embedded within epoxy [63], as illustrated in the left inset of Figure 3A. Macroscopic architectures, such as a honeycomb composite structure 
(left image of Figure 3A), can achieve specific and anisotropic properties to enable mechanical strength and toughness with significantly less weight. The printing of such fiber-reinforced epoxy inks could be used to create structures with exceptional toughness, impact resistance and Young's moduli that are factors of 10-20× greater than common commercial 3D printable inks, as shown in the graph of Figure 3A [63].

Second, lightweight structures can be created by first printing polymer templates followed by subsequent coating of metals [145] or ceramics [146]. For example, 3D printing of polymer lattices via the removal of a sacrificial template leaves a hollow wood-pile structure coated with silicon (Figure 3B) [147]. As shown in the bottom inset of Figure 3B, a tri-layer $\mathrm{Si} / \mathrm{SiO}_{2} / \mathrm{Si}$ tube with a wall thickness of $300 \mathrm{~nm}$ can be produced. However, high temperature chemical vapor deposition (CVD) processes are required, which may not be compatible with the co-printing of biological structures or low-temperature polymer substrates. Nevertheless, such designs suggest that 3D printing provides the ability to create features that are smaller than the printing resolution.

Third, the ability to create freeform architectures without the constraints of conventional planar fabrication processes also provides an opportunity for utilizing numerical analyses in the structural designs to fine-tune 3D architectures and properties. For instance, auxetic materials with enhanced shear and indentation resistances can be created via the combination of 3D printing with topology optimization. Clausen et al. have shown the 3D printing of architectures with Poisson ratios of 0.8 that were designed via numerical optimization [148]. In another example, Shan et al. demonstrated 3D printed multi-stable structures with enhanced absorption of compression energies, as shown in Figure 4C [149]. The printed beam elements are able to respond to external loading by reconfiguring, in a reversible fashion, into other stable structures without mechanical 
failure. Such novel geometries could potentially enable the creation of robust bionic devices with enhanced impact resistance and energy-absorbing capabilities to withstand wear and impact.

Finally, beyond serial printing using individual nozzles, a more seamless and parallel merging of multiple materials can be achieved by incorporating nozzle designs that enable the co-printing of different materials to achieve unique multi-material architectures. In addition to increasing throughput [150], multi-nozzle microfluidic printheads allow for the rapid co-printing of two different viscoelastic inks to create architectures with sharp transitions of different materials [60]. This multi-material integration has also been demonstrated via an active mixing of viscoelastic inks to achieve programmable control over local compositions [59].
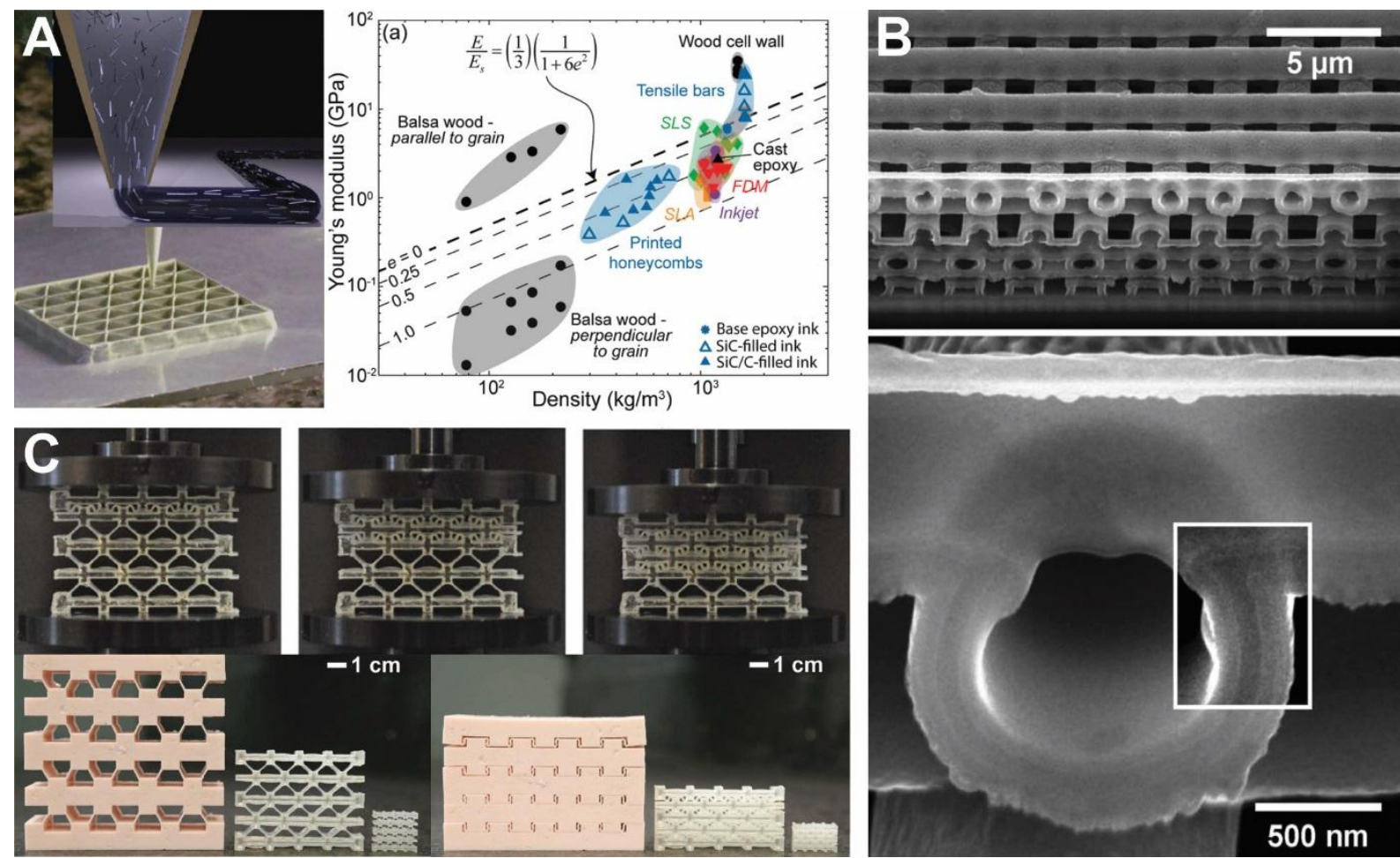

Figure 3. 3D printing can create macroscale architectures exhibiting interesting mechanical properties. (A) 3D printing of lightweight cellulose composite. The inset illustrates the alignment of high aspect ratio fillers inside the nozzle (left figure). A plot of Young's modulus vs. density of 
3D printed balsa wood and 3D printed tensile bars with fillers show a factor of $10-20 \times$ higher longitudinal Young's moduli compared to most commercially available 3D printed polymers [63]. (B) Hollow-wood pile structure, where the higher magnification SEM image (bottom image) shows a tri-layer $\mathrm{Si} / \mathrm{SiO}_{2} / \mathrm{Si}$ tube wall [147]. (C) Multi-stable architected materials, where the top sequential images demonstrate that the structure retains a deformed shape after removal of a vertical load. Left and right bottom images show the structures before and after compression, respectively [149]. Reprinted with permission from Refs. [63], [147], [149], respectively. Copyright 2014 John Wiley \& Sons, 2006 John Wiley \& Sons, 2015 John Wiley \& Sons.

\section{D Printing of Spatiotemporal Biomolecular Gradients}

The ability to mimic the dynamic microenvironment surrounding cells in natural tissues is critical to engineering the biotic/abiotic interfaces found in bionic systems [151-153]. Indeed, cell fate is influenced by numerous molecular factors and interactions that require meticulous control for the regeneration of functional tissue $[154,155]$. In order to achieve such control, engineered matrices should ideally be capable of generating multiplexed spatiotemporal molecular gradients. Extensive research efforts to engineer such matrices have resulted in a number of promising methods to generate and control molecular gradients (Figure 4). These methods can generally be categorized as static $3 \mathrm{D}$ methods (Figure $5 \mathrm{~A}$ ) where the gradient is fixed once programmed, and dynamic 4D methods (Figure 5B) where the gradients can be reprogrammed by the user. The focus of this review is on strategies to generate biomolecular gradients that are compatible with 3D printed systems, and as such a complete description of tissue culture compatible methods to generate biomolecular gradients is beyond the scope of this review. The reader is referred to several literature sources that provide excellent in-depth discussions of such methods $[151,155,156]$. 


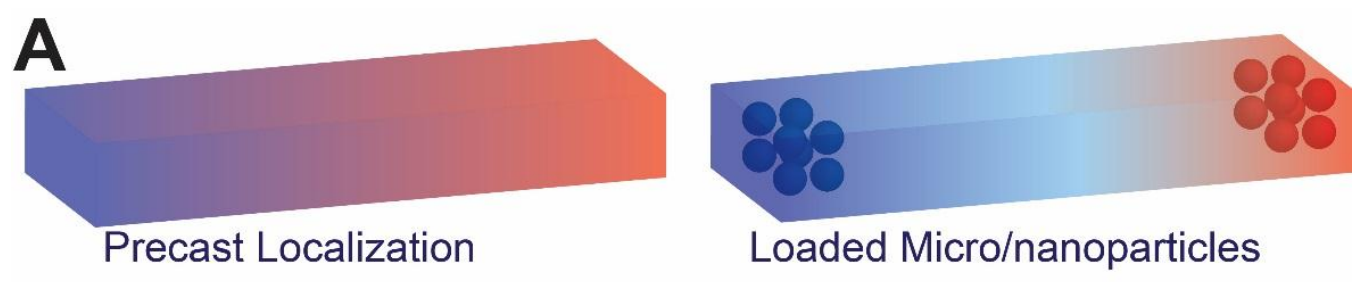

\section{Static 3-D Methods}

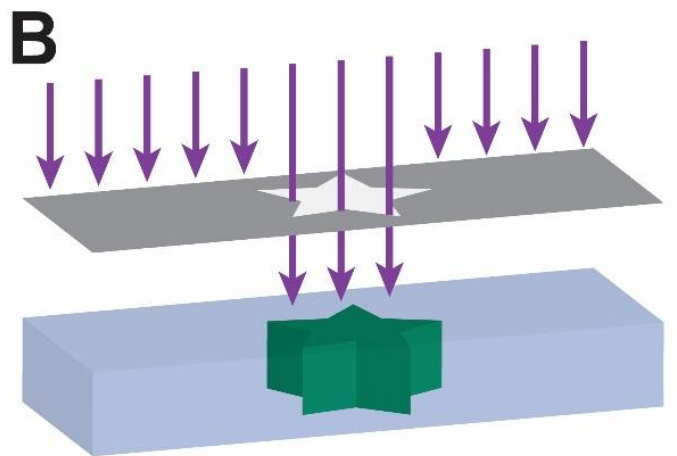

Photodegradable Crosslinks

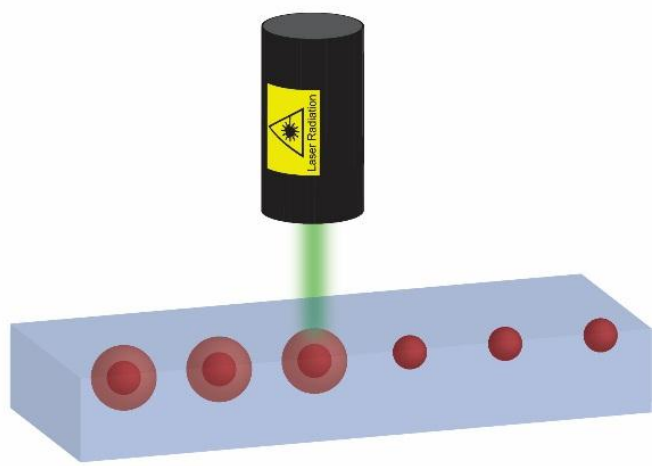

Stimuli-Responsive Capsules

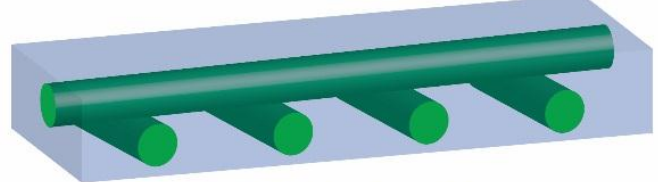

Microfluidic Networks

\section{Dynamic 4-D Methods}

Figure 4. Schematic of 3D static and 4D dynamic printing methods to create chemical and biomolecular gradients. (A) Static methods allow for a preprogrammed gradient to be developed, typically based on passive diffusion from payload depots. (B) Dynamic methods allow for "on the fly" active reprogramming of gradients, by including the fourth dimension of time.

\section{D Static Printing of Gradients}

The simplest method for creating a hydrogel scaffold with a static biomolecular gradient is through direct spatial localization of biological factors within the hydrogel. In this approach, once the spatial gradient has been generated, the temporal persistence of the gradient is based solely on the 
diffusivity of the biomolecule within the hydrogel, which is generally a function of the size and chemistry of the biomolecule, crosslink density and water fraction of the hydrogel, and molecular interactions between the biomolecule and the hydrogel polymer. Scaffolds with such gradients can be made via solid freeform fabrication through sequential deposition of hydrogels containing various concentrations of differing biomolecules. This strategy is compatible with both extrusion and light-based printing methods [157]. These approaches are suitable for creating relatively simple gradients using a few biomolecules, and gradients of soluble factors typically persist for days. Applications where a gradient is desired for longer durations (weeks to months) require strategies to immobilize the biomolecule on the hydrogel polymer.

An alternative strategy to creating hydrogel gradients is to apply the gradient after the hydrogel scaffold has been formed. A common approach to doing this is inkjet printing of biomolecule solutions onto a hydrogel substrate [158-162]. As the solution droplet impacts the surface of the hydrogel, it is quickly absorbed and the biomolecules are localized to the 2D footprint of the droplet. Gradients arrays can be created by dispensing varying numbers of droplets over the same area. The precise control over droplet volumes in inkjet printing allows researchers to create gradients with precise concentrations and varying gradient profiles. Nuzzo et al. have developed a variant of this method [163]. By using soft contact printing of a microfluidic network with a permeable membrane, they were able to demonstrate transfer printing of complex gradients into the hydrogel substrate. Although these methods are most commonly utilized with flat hydrogel substrates, they can easily be integrated with methods for freeform fabrication of hydrogel scaffolds by inkjet or transfer printing of gradients at select hydrogel layers during the freeform fabrication process. 
The simplicity of directly incorporating gradients in the hydrogel has made this approach widely used, particularly in fundamental studies where the effect of a particular gradient on cell behavior is being studied. However, a major consideration is the lack of control over the temporal evolution of the gradient and the cumbersome nature of incorporating multiplexed gradients. Micro/nanoparticles loaded with biomolecules represent a versatile approach to delivering multiplexed gradients with additional control over the release kinetics $[164,165]$. While such particles can be made via numerous methods and from a wide range of materials, they are most commonly formulated from biodegradable polymers using double emulsification or coacervation methods [166]. The particles can be efficiently loaded with a variety of biomolecular payloads, while maintaining their activities [167].

Synthesizing particles loaded with different factors and localizing them within a hydrogel matrix can lead to the generation of multiplexed spatial gradients. The payload release kinetics can be adjusted by controlling the particle properties (e.g., diameter, shell thickness, porosity, etc.). However, spatiotemporal control over the gradients is typically coarse, as most scalable methods to synthesize particles result in highly polydisperse populations. Fine control over release dose is often required, and recent research efforts have focused on achieving robust control over release kinetics. To this point, numerous groups have developed microfluidic methods to synthesize monodisperse particles with a high degree of control over particle properties [34,168,169]. While it is possible for such monodisperse particles to first be synthesized and collected, and then to be formulated into an ink for 3D printing, a more natural solution is for direct incorporation of the microfluidic devices in the printing nozzle. Currently, research efforts to develop microfluidic print nozzles have demonstrated single nozzle printing of multiple materials with varying 
mechanical properties, with the added ability to create discrete or graded interfaces between the materials [60].

We have developed an alternative strategy to 3D print highly monodisperse capsule arrays (Figure 6A) [62]. First, aqueous cores are printed onto a hydrophobic substrate. Next, the core is encapsulated by dispensing a biocompatible polymeric solution which rapidly evaporates, leaving a solid polymer shell. The versatility of this approach is the ability to accurately dispense multiplexed arrays over large areas with precise control over the core composition and the shell thickness. Figure 5B shows a 'tiger' consisting of 4,000 red and blue cores, with a spacing of 400 $\mu \mathrm{m}$. Adjacent is an optical image of a $\mathrm{pH}$ gradient array generated by printing varying volumes of an acidic and basic ink. The color of each drop is a result of the $\mathrm{pH}$ indicator $\mathrm{m}$-cresol purple. The shell thickness of the capsules was varied by adjusting the concentration of the polymer in the dispensed shell solution. In this manner, we were able to realize control over the passive release kinetics of horseradish peroxidase (HRP) from the capsule core.

The passive 3D methods described here to generate biomolecular gradients could represent powerful tools for studying the impact of a surrounding microenvironment on a wide range of cellular responses. The major advantage of these methods is their broad applicability to a wide range of biomolecules and hydrogel systems. For example, chemical modification of the biomolecules and hydrogel polymers is generally unnecessary. The limitation, however, is that the temporal evolution of the gradient is ultimately controlled simply by diffusion of the biomolecules through the hydrogel matrix or capsule shell. As such, there are limited opportunities to tailor the temporal response, especially in the case of multiplexed gradients. A more compelling alternative is to introduce vehicles for achieving precision control over time as well as space. 


\section{D Dynamic Printing of Gradients}

The incorporation of a selective, stimuli-responsive element in a biomolecular gradient can provide an additional level of control over its temporal evolution. The concept of stimuli-responsive controlled release has been extensively explored for applications in drug delivery. Capsules, nanoparticles, and hydrogels have been developed that release a chemical payload in response to a wide range of user-applied external stimuli such as light, heat and magnetic field, or internal biological stimuli such as $\mathrm{pH}$, temperature and biomolecular signaling [170-173]. The main advantage of stimuli-responsiveness in drug delivery is the ability to specifically deliver drugs to affected cells and tissues while minimizing side effects due to interactions with healthy tissues and cells. In tissue engineering and the creation of bionic devices, the primary advantage of stimuliresponsive controlled release is the ability to reprogram gradients in response to changes in cell growth, differentiation, and/or migration.

Novel labile chemical linkers have been used to pattern and release biomolecules from polymer backbones in response to external stimuli. Most commonly, a photolabile linker is used to tether the biomolecule to the hydrogel polymer [174]. When exposed to a particular wavelength of light, the linker is degraded and the biomolecule becomes soluble. The gradient can be established by either the soluble or insoluble fraction of the biomolecule depending on the activity of the particular system. Spatial patterning can be achieved by shining light through a photomask, or by using a two-photon response where the linker is only degraded in the highly focused region of a laser beam. While this method provides excellent spatiotemporal control, selective multiplexed release requires engineering orthogonal linkers for each molecular factor to be released, which can become a major technical challenge. Additionally, the activities of many biomolecules can be decreased due to the covalent linking, and strategies to mitigate this can be challenging. 
We have demonstrated a novel, selectively photoresponsive system using our 3D printed capsule platform [62]. In order to make the capsules photoresponsive, we loaded the shells with gold nanorods. The LSPR wavelength of the nanorod is strongly dependent on its length. In response to light exposure at the LSPR wavelength, the nanorods are rapidly heated, melting the polymeric shell, such that the capsule ruptures - quickly releasing the payload. Figure $6 \mathrm{C}$ shows the release of horseradish peroxidase from 2D printed capsules that have been ruptured. In this system, selective multiplexed release can be easily achieved, as no covalent modification is required to encapsulate the biomolecules, and gold nanorods with varying LSPR wavelengths can be routinely prepared in large quantities.

Microfluidic channels have been directly incorporated in hydrogels, providing a means to flow biomolecule solutions through gels as another strategy for creating dynamic gradients $[175,176]$. Gradients can be established in two ways. First, a gradient can be generated by the diffusion of biomolecules out of the microfluidic channel into the hydrogel matrix. Second, the flow of multiple solutions through a designed gradient generator can create precise gradients within the channel. This allows for the flexibility of generating steady-state gradients that can be maintained over long periods of time. Additionally, the concentrations of solutions can be rapidly varied for precise control of the temporal delivery of biological factors. However, multiple independent networks are required for multiplexed gradients, and an extensive pumping and fluid-handling infrastructure is required. This approach is thus more suitable for generating vasculature by consistently supplying nutrients to and removing waste from the tissue, rather than a means of generating transient gradients of biomolecules that can control cell fate at a local level. 

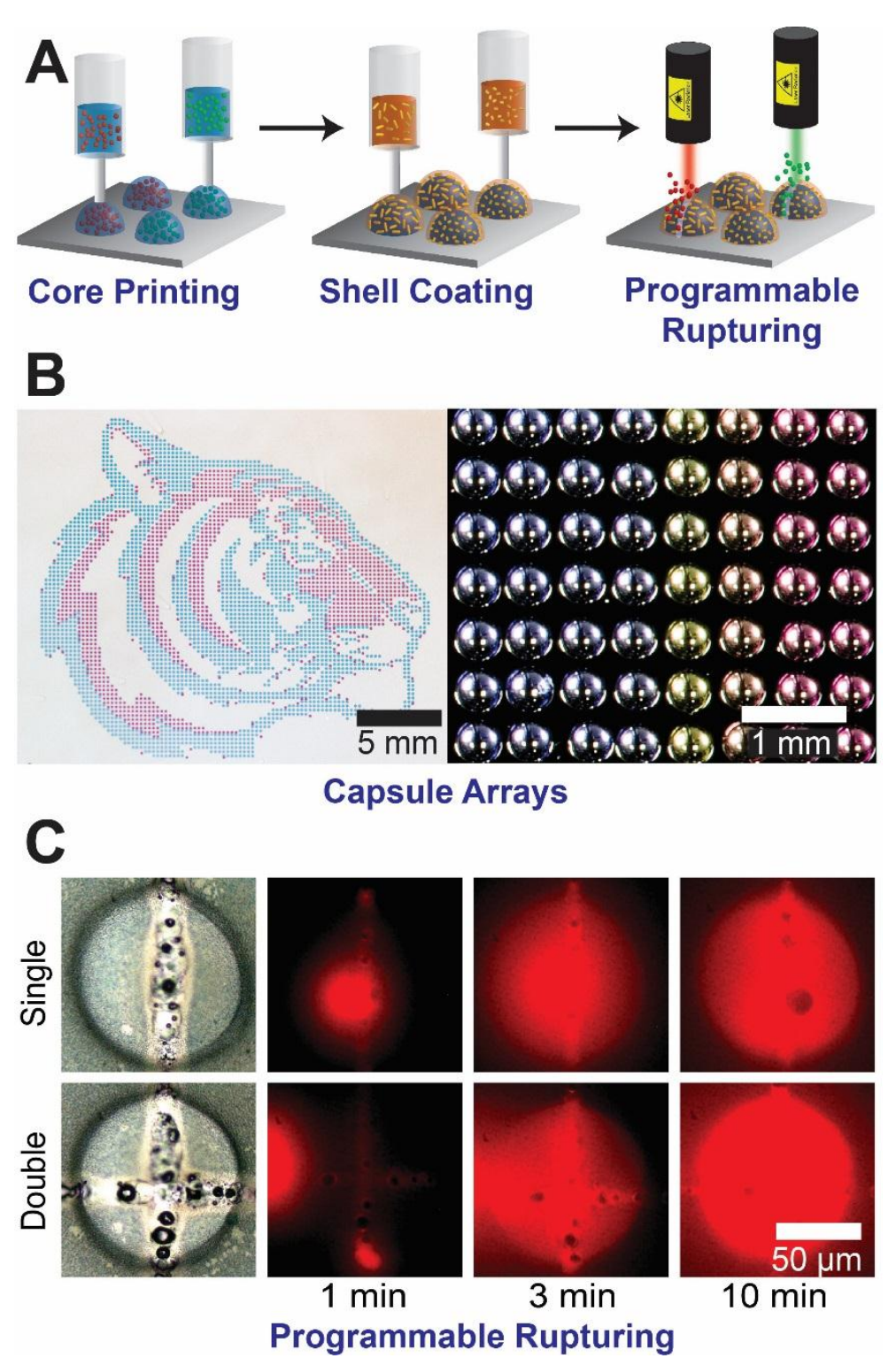

Figure 5. (A) Schematic showing a 3D printing strategy to creating stimuli-responsive capsules that can be selectively ruptured to release payloads in response to optical stimuli. Incorporation of gold nanorods in the shells allows the capsules to be ruptured by exposure to laser wavelengths determined by the lengths of the incorporated nanorods. (B) Optical images of complex capsule arrays including a printed 'tiger' and a $\mathrm{pH}$ gradient array with different colors from an indicator dye. (C) Programmed rupture and release of HRP from capsules by selective laser exposure [62] Reprinted with permission from Ref. [62]. Copyright 2015 American Chemical Society. 


\section{D Hybrid Systems with Dynamic Gradients}

A longstanding challenge in tissue engineering has been the incorporation of dynamic gradients within macroscale 3D scaffolds. A significant hurdle to accomplishing this goal is the inherent challenges of multi-material 3D printing. Stimuli-responsive capsules and microfluidic networks are strategies for generating dynamic gradients that require novel materials and processes in order for them to be compatible with $3 \mathrm{D}$ printing. In order to $3 \mathrm{D}$ print complex arrays of stimuli responsive capsules, we have developed a new type of ink based on a water-in-oil emulsion [62,177-179]. The emulsion inks were prepared via high-speed dispersion of aqueous biomolecule solutions into a non-polar polymer solution. The emulsion-based ink was then directly printed into a thin layer of an aqueous hydrogel. Once printed, the solvent rapidly evaporates, leaving behind a solidified capsule sealed within the hydrogel. Thus, the hydrogel and capsules could be readily printed in a layer-by-layer fashion to create complex 3D hierarchical programmable capsule arrays. Since the 3D printing process is based on digital software control, rationally designed advanced architectures could be constructed. For instance, Figure 6A (left) shows an optical photograph of a hollow hydrogel cylinder containing alternating layers of red and blue capsules in the cylinder wall, and (right) two inverted pyramidal arrays of capsules printed within a solid hydrogel cube.

Several recent examples have demonstrated the freeform fabrication of microfluidic networks in macroscale hydrogel scaffolds [177-179] via the use of a sacrificial ink to define the channel lumen. The primary challenge in printing 3D microfluidic networks is that the sacrificial ink and hydrogel scaffold cannot be printed in a layer-by-layer manner, as doing so would not allow for continuous channels in the Z-direction of the scaffold. Bhattacharjee et al. have demonstrated the use of a granular gel medium into which highly complex continuous 3D channels could be printed (Figure 6B) [177]. Particle jamming in the media allowed for a system where the yield stress was 
high enough to prevent disruptions in the printed network due to differences in densities of the gel media and vascular network. Yet, the yield stress was sufficiently low to allow the print nozzle to travel through the media without leaving a void in its wake.

In contrast to the previous example, another approach to creating microfluidic networks first involves the printing of a sacrificial ink in the shape of the desired network. Then, the scaffold is deposited around the solidified network. Finally, once the scaffold is solidified the sacrificial network is removed. Miller et al. demonstrated such an approach utilizing a carbohydrate-based glass as the sacrificial ink (Figure 6C) [179]. The carbohydrate glass is printed in the molten state and allowed to cool and solidify. Once the gel scaffold is cast and solidified, the carbohydrate glass is dissolved away in water. A key advantage of this is the compatibility of the sacrificial ink with a wide range of hydrogel polymers. In another similar example, Kolesky et al. utilized inverse temperature responsive gelation of a sacrificial ink and hydrogel scaffold to create 3D microfluidic networks (Figure 6D) [178]. Both the sacrificial ink (Pluronic ${ }^{\circledR}$ F-127) and hydrogel scaffold (gelatin methacrylate) are fully gelled between $4-22^{\circ} \mathrm{C}$. The F-127 liquefies below $4{ }^{\circ} \mathrm{C}$, whereas, the gelatin is liquid above $22{ }^{\circ} \mathrm{C}$. Thus, to create a microfluidic network the F-127 is first printed into the shape of the channels above $4{ }^{\circ} \mathrm{C}$. Next, above $22{ }^{\circ} \mathrm{C}$ the liquid gelatin is poured around the F-127 network and cooled to allow it to solidify. Finally, the temperature is lowered below 4 ${ }^{\circ} \mathrm{C}$ to liquefy the F-127, leaving channels in the gelatin scaffold. These examples show the wide diversity in materials and processes that can be utilized to generate systems with dynamic and complex biomolecular gradients. 

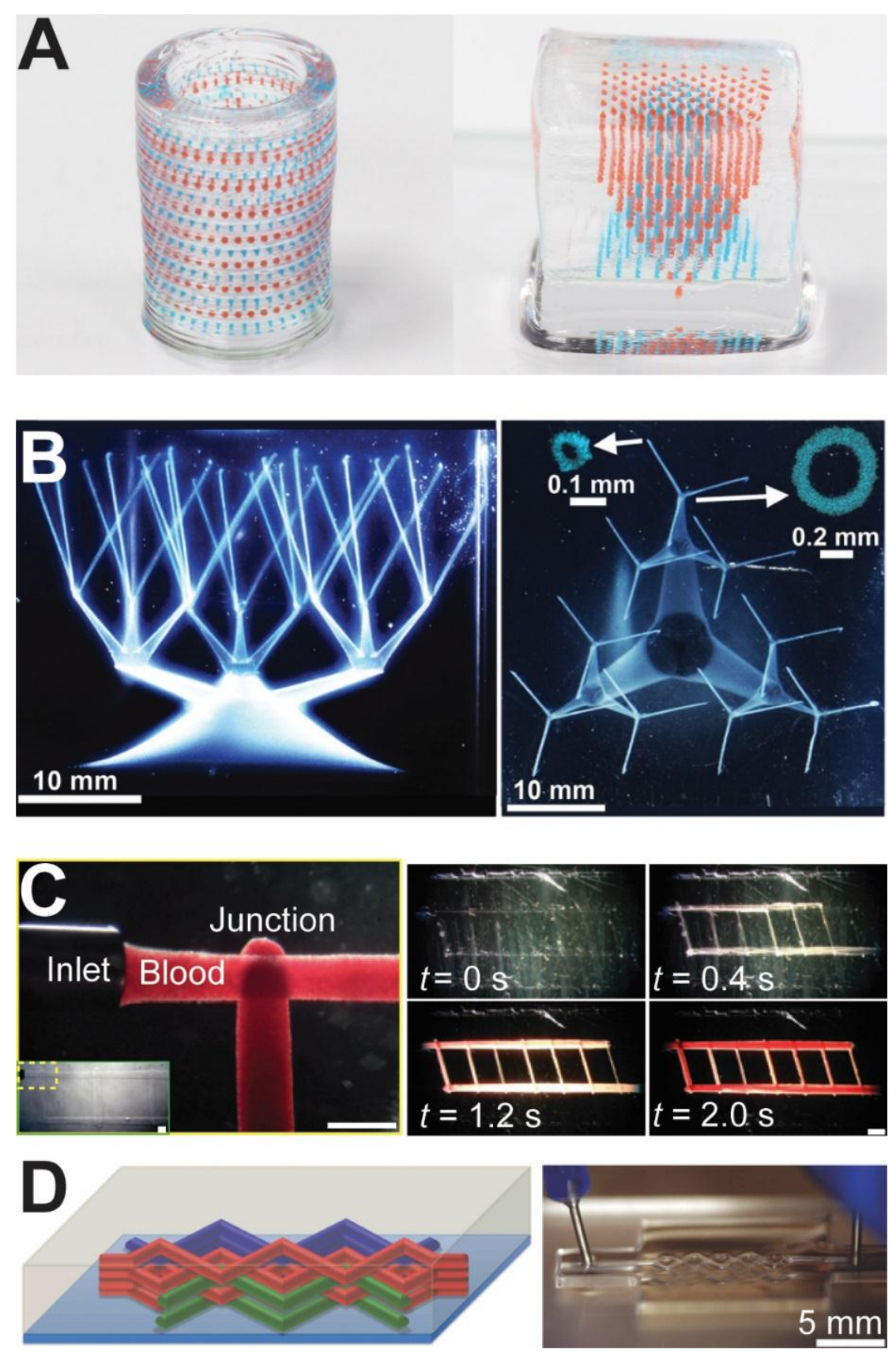

Figure 6. 3D printing strategies to create gradients in macroscale structures. (A) An emulsion printing strategy to create stimuli-responsive multiplexed arrays of capsules within 3D hydrogel matrices [62]. (B) Direct printing of vascular networks in granular media. Jamming of the media allows the printed network to be stabilized as it is printed [177]. (C) A carbohydrate glass is printed as a sacrificial scaffold for the vascular network. Once the gel matrix is cast, the scaffold is 
dissolved leaving behind open channels [179]. (D) In this example, the authors use a fugitive ink to create microfluidic channels in a hydrogel. After the channels are formed, the ink is removed by decreasing the temperature to fluidize the fugitive ink [178]. Reprinted with permission from Refs. [62], [177], [179], [178], respectively. Copyright 2015 American Chemical Society, 2015 American Association for the Advancement of Science, 2012 Nature Publishing Group, 2014 John Wiley \& Sons.

\section{D Printed Anatomical Design}

3D printed multi-scale biological systems have included artificial tissues, organs, biomedical implants, and bionic tissues. To date, 3D printed biological systems have been approached from three primary design paradigms, which include: (1) anatomical design, (2) mechanical (i.e. physical or topological) design, and (3) biochemical design. Anatomical design involves the development of 3D printed materials and devices which match the inherent anatomical structures by mimicking their 3D geometry. Some of the earliest efforts in anatomical 3D printed biological systems came in the form of engineering studies for craniofacial bone regeneration. In one such study, helical computed tomography (CT) images acquired from a dry mandible were used to generate the resultant 3D models for the printed scaffolds [180]. It was shown that the dimensional error of the 3D printed anatomical part, here the mandibular anatomy, varied from approximately 1-3\% depending on the printing technique used. Recently, anatomical design of 3D printed biological systems has expanded to include: (1) heart tissues [181], (2) nerve scaffolds [64], (3) vascularized bone grafts [182], and (4) artificial skin [183].

Anatomical structures include external and internal tissues and organs which range from micrometer to centimeter length scales. For example, as shown in Figure 7A, 3D printing has fabricated biomimetic tri-leaflet heart valve conduits, which use human aortic valvular interstitial 
cell-laden hydrogels of methacrylated hyaluronic acid and gelatin [181]. Another highlight of anatomical design was recently demonstrated in the form of 3D printed anatomical nerve regeneration pathways. As shown in Figure 7B, 3D models developed from structured light scanning of nerve tissue enabled the regeneration of complex bifurcating peripheral nerves in rats [64]. In addition to 3D printed scaffolds for heart and nerve tissue, advances in 3D printed bone and vasculature engineering have shown the ability to incorporate vasculature within anatomical bone grafts for potential connection of the tissue to the blood supply (Figure 7C) [182]. As discussed above, not only are anatomical design principles driving the manufacturing of multiscale anatomical internal tissues and organs $[55,64,67,178,181,184]$, but they are also guiding the exploration of external tissues which interact with the surrounding environment [183]. For example, Figure 7D shows that 3D printed artificial skins can be realized which contain microstructured biomimetic surface topographies that achieve innovative hydrodynamic flows [183]. 

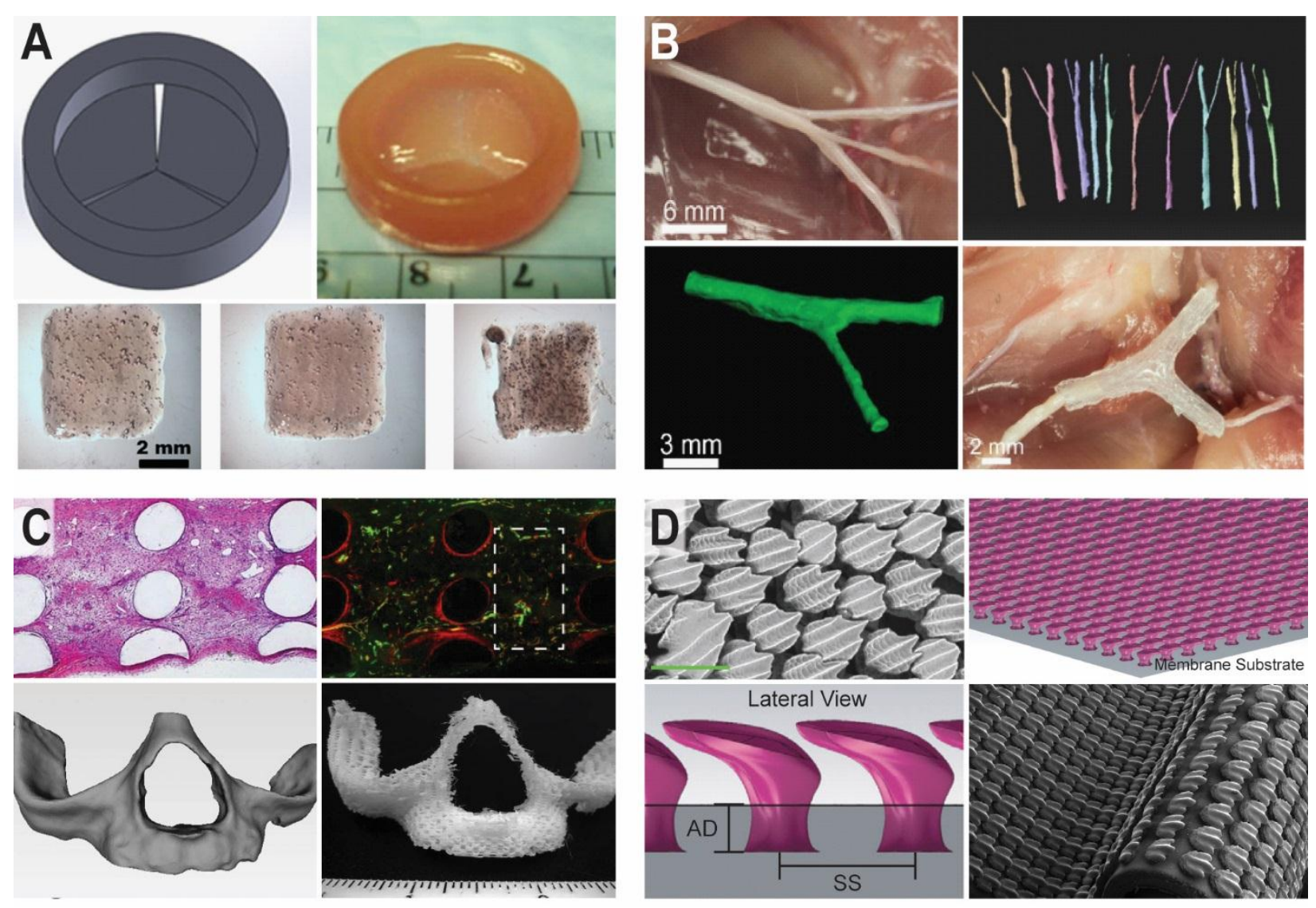

Figure 7. 3D printed anatomical design strategies. (A) 3D printed tri-leaflet heart valve [181]. (B)

3D printed anatomical nerve regeneration pathway [64]. (C) 3D printed vascularized bone architectures [182]. (D) 3D printed biomimetic artificial skin [183]. Reprinted with permission from Refs. [181], [64], [182], [183] respectively. Copyright 2014 Elsevier, 2015 John Wiley \& Sons, 2014 John Wiley \& Sons.

\section{D Printed Biomechanical Design}

Mechanical (or topological) design involves the mimicry of native mechanical properties and cues, such as stress-strain behavior, topographical structures, and microstructures, in 3D printed scaffold architectures. Some of the earliest efforts to examine the influence of physical cues in 3D printed biological systems included the design of porous scaffolds for bone engineering applications [185]. 
For example, 3D printing was used to assemble hydroxyapatite scaffolds for bone regeneration over a range of mechanical properties, such as porosity and strength. It was shown that the mechanical properties and biological consequences of 3D printed scaffolds for bone regeneration may be tuned by controlling the wall and channel thicknesses of 3D printed grid-based scaffolds. Recently, mechanical design of 3D printed biological systems has expanded to include: (1) 2D topographical cues in biomimetic in vitro models [65], (2) 3D topographical cues in 3D tissue scaffolds [64], and (3) bio-inspired mechanical systems [186].

The geometry of physical cues has included channels, grooves, and filaments. As shown in Figure $8 \mathrm{~A}$, we recently demonstrated that a biomimetic nervous system on a chip technology can be realized via the controlled guidance of axons within 3D printed polycaprolactone microchannels [65]. Similarly, Figure 8B shows that 3D printed physical cues in the form of microgrooves in anatomical elastomeric scaffolds for peripheral nerve regeneration resulted in the aligned growth of axonal networks and cytoskeletons of Schwann cells [64]. The parallel and orthogonal assembly of material filaments has provided flexible mechanical design opportunities in terms of controlling scaffold strength and fate of interacting cellular components. Further, the ability to control filament assembly in non-uniform geometric patterns other than layer-by-layer approaches also enables one to mimic various biomechanical systems. For example, as shown in Figure 8C, it was recently shown that radial and spiral filament strengths control the loading response in natural webbed systems, here a spider's web [186]. 


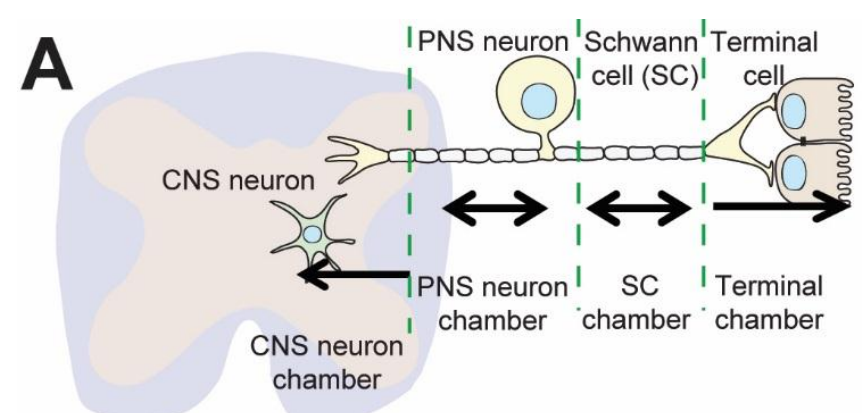

PNS neuron chamber
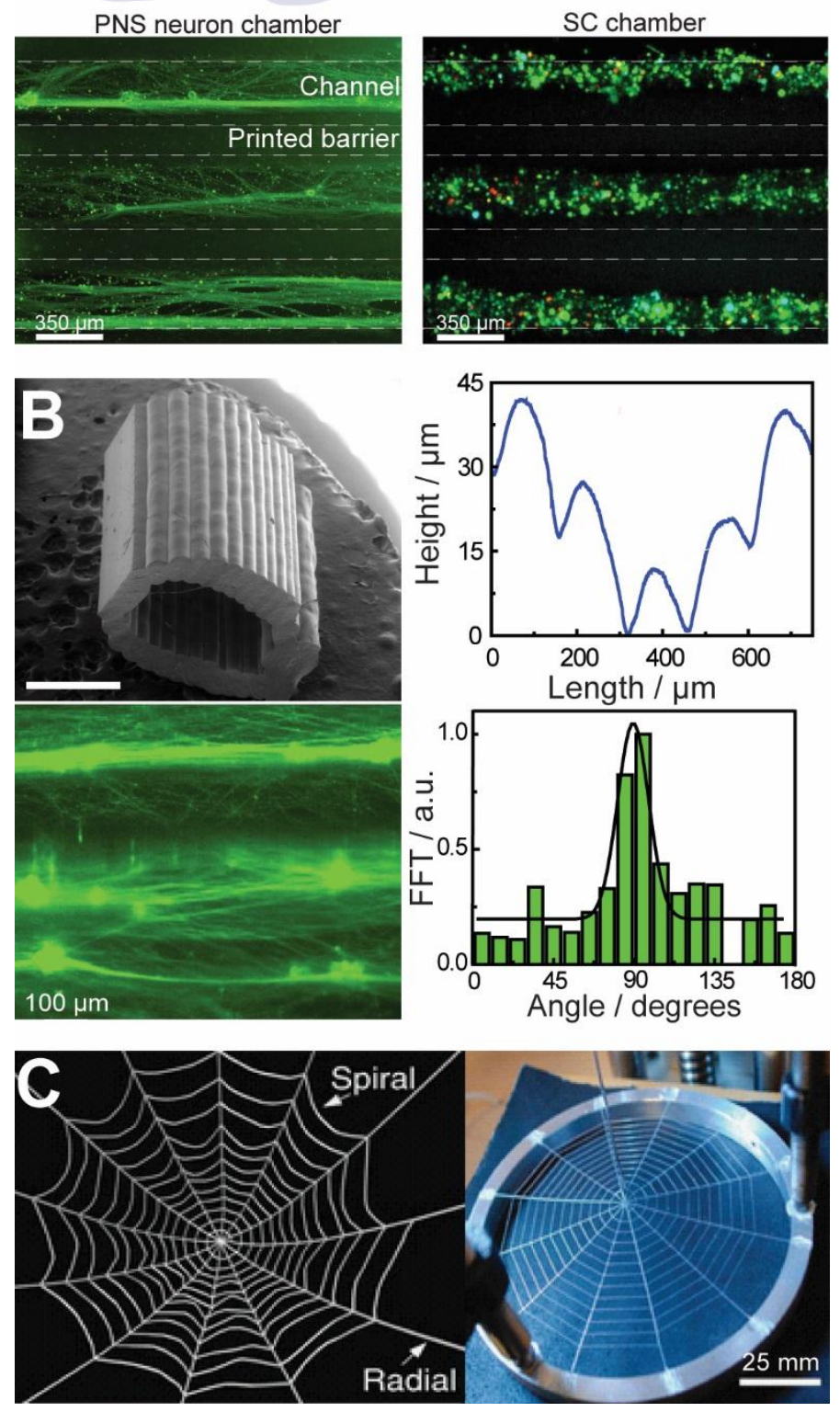

Figure 8. Mechanical design methodologies in 3D printed biological systems. (A) 3D printed microchannels control the growth of axonal networks in a 3D printed nervous system on a chip [65]. (B) 3D printed microgrooves in elastomeric anatomical nerve guides control the alignment 
of the regenerating axonal network longitudinally toward the injury site [64]. (C) 3D printed spider web displaying interacting radial and spiral elastomeric filaments [186]. Reprinted with permission from Refs. [65], [64], [186], respectively. Copyright 2015 Centre National de la Recherche Scientifique (CNRS) and Royal Society of Chemistry, 2015 John Wiley \& Sons, 2015 Nature Publishing Group.

\section{D Printed Biochemical Design}

Biochemical design involves the mimicry of a native biochemical structure or profile, which may include either uniform or non-uniform distributions of biochemical factors. Some of the earliest efforts included the patterning of protein gradients on 2D substrates [158]. In this study, inkjet printing was used to print ciliary neurotrophic factor (CNTF) gradients and examine their effect on the multi-potency and differentiation of neural stem cells (NSCs) [158]. It was shown that NSCs cultured on a printed gradient of increasing levels of CNTF showed a linear increase in the numbers of cells expressing glial fibrillary associated protein (GFAP), demonstrating a functional 3D printed gradient of CNTF on a 2D substrate. As discussed, 3D printing also has the ability to apply anatomical $[64,67,178,181]$ and mechanical $[64,65,186]$ design principles toward the manufacturing of novel biological systems. Thus, the multiscale nature of the approach which combines anatomical, mechanical, and biochemical design paradigms also allows for the integration of biochemical gradient technologies within macroscale biological architectures in order to realize novel platform technologies and biomedical devices. In our study of nerve regeneration in bifurcating mixed nerve pathways, 3D printing was used to incorporate multicomponent biochemical gradients of nerve growth factor (NGF) and glial cell-derived neurotrophic factor (GDNF) within a global scaffold architecture, to support the regeneration of 
both motor and sensory nerve pathways (Figure 9A) [64]. As shown in Figure 9B, scaffolds containing path-specific multi-component gradients led to regenerated tissue which possessed enhanced functional return relative to scaffolds which did not contain supplemented gradients, thus validating the potential for functional 3D printed multi-component gradients within a scaffold architecture to improve regenerative outcomes. 

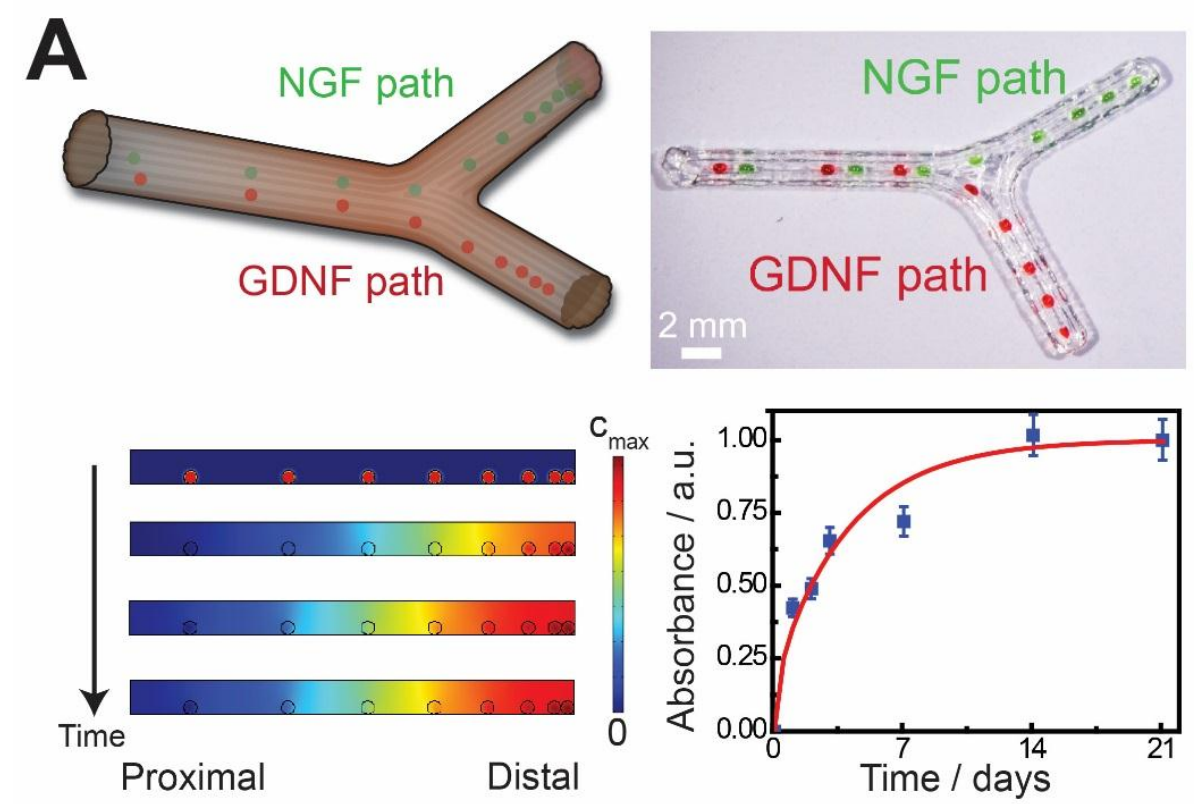

B
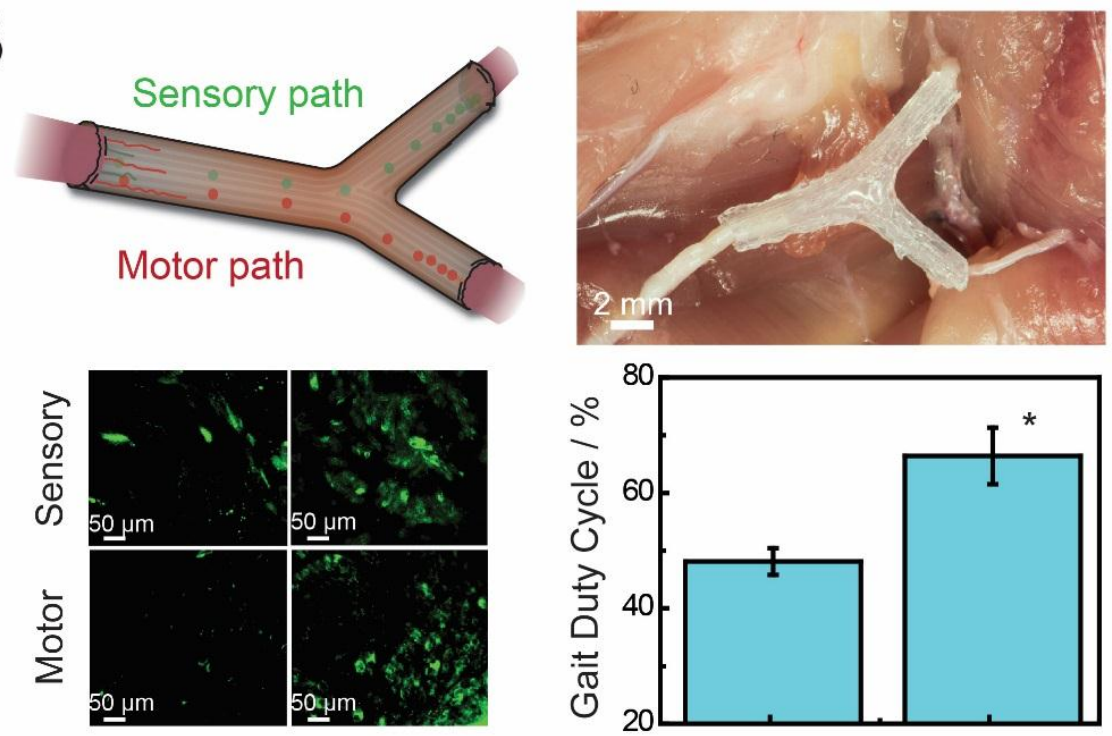

(-) Gradients (+) Gradients

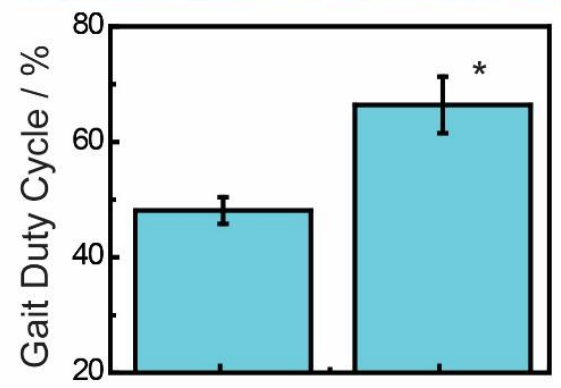

(-) Gradients (+) Gradients

Figure 9. Biochemical design strategies in 3D printed biological systems. (A) Path-specific 3D printed multi-component gradient in anatomical nerve regeneration pathways [64]. (B) Effect of the functional 3D printed path-specific regeneration on the regeneration of motor and sensory nerve pathways, and the functional return of complex regenerated peripheral nerve injuries [64]. Reprinted with permission from Ref. [64]. Copyright 2015 Royal Society of Chemistry. 


\section{D Printed Conducting Ink Electronics}

Modern prosthetics typically incorporate electronics to mimic, restore, and/or augment the complex functionalities of biological constructs $[1,12,18,19,21,22]$. The ability to create advanced electronics with 3D printing could lead to methods for directly printing both the mechanical prosthetics and the incorporated electronics from the same machine, replete with customization of both geometries and functionalities. This section focuses on the printing of conducting nanoscale inks such as metal nanoparticles, which can act as interconnects, or can be made into passive devices such as strain sensors and antennas. For instance, Ahn et al. described the synthesis of a highly concentrated (> $70 \mathrm{wt} \%)$ viscoelastic ink with silver nitrate, poly(acrylic acid) and diethanolamine [90]. Compared to previously published inkjet printed metal nanoparticle inks, this ink formulation has a key advantage in its ability to create three-dimensional interconnect arches as shown in the inset of Figure 10A. These features overcome the conventional planar constraints of traditional printing methods, allowing for interconnects that can span and stretch across circuit elements in three dimensions [90]. Further, Ahn et al. demonstrated an impressive minimum feature size of $2 \mu \mathrm{m}$ as shown in Figure 10A. This enabled the creation of features such as a transparent conductive grid [187]. Significantly, the resistivity $\left(5.2 \times 10^{-7} \Omega \cdot \mathrm{m}\right)$ approaches the resistivity of bulk silver $\left(10^{-8} \Omega \cdot \mathrm{m}\right)$, and this value can be achieved by using a relatively mild annealing process $\left(250^{\circ} \mathrm{C}\right)$ for a short time $(30 \mathrm{~min})$. The formulation of the inks and the printing of the electrodes into wavy architectures enabled the printed conductors to withstand repeated stretching and bending (maximum strain of 25\%). Such attributes are important in the creation of stretchable bionic devices such as skin sensors [12].

The ability of extrusion based $3 \mathrm{D}$ printing to accommodate diverse materials with a wide range of viscosities allows for the incorporation of classes of electronic materials that are incompatible with 
other patterning processes, such as inkjet printing or dip-pen lithography [188]. For instance, eutectic gallium-indium alloy (EGaIn) [189] is a highly conductive $\left(3.4 \times 10^{4} \mathrm{~S} \mathrm{~cm}^{-1}\right)$ liquid metal that has recently been explored as a 3D printable conductor [70,190]. Intriguingly, despite its liquid nature at room temperature (melting point $=15.5^{\circ} \mathrm{C}$ ), mechanically stable three-dimensional structures can be achieved. This is due to a high surface tension $\left(0.6 \mathrm{~N} \cdot \mathrm{m}^{-1}\right)$ thin oxide film that is formed on the surface of the liquid (Figure 10B). Further, a printing resolution of $100 \mu \mathrm{m}$ has been demonstrated, and unlike most sintered solid metal, the liquid allows for the creation of highly stretchable (up to $100 \%$ elongation) electrodes when encapsulated within an elastomeric polymer [190], enabling the creation of stretchable devices such as strain gauges. Given the work function of $-4.2 \mathrm{eV}, \mathrm{EGaIn}$ has also been shown to be a suitable printable cathode. This is particularly useful for low melting point polymeric 3D printed constructs, as thermal sintering processes can be avoided [61].

The ability to pattern in three dimensions allows for the creation of electronics with unique or unusual performances in comparison with planar patterning techniques. For instance, the printing of silver nanoparticles on a three-dimensional construct allowed for the fabrication of an antenna with an order of magnitude improvement over monopole antenna designs (Figure 11C) [191]. Further, the co-printing of conductive traces with an elastomeric substrate $[59,60]$ can result in the freeform fabrication of three dimensional structures containing electronic components. For instance, capacitive soft strain sensors can be realized via the printing of core-shell fibers with silicone and conductive fluids [71]. As shown in Figure 10D, the embedded 3D printing of conductive carbon grease within an elastomeric polymer enabled the seamless fabrication of complex arrays of strain sensors within a glove, that can be used to monitor the motion of a user's hand. Indeed, these demonstrations highlight the many distinct advantages of 3D printing in the 
fabrication of electronic devices. These include the realization of devices customized to conform to the user's morphology, and customization of functionality by incorporating different classes of materials to create functional components within soft and stretchable constructs.

The bottom-up nature of 3D printed electronics - and the exclusion of the harsh chemicals and temperatures found in microfabrication processes - allows for the co-printing of electronics with biological materials to yield novel constructs even including bionic organs $[55,67]$. This threedimensional interweaving of electronics and biology using a multi-material 3D printing process was demonstrated by our group. Specifically, the co-printing of a cell-laden scaffold and conductive traces enabled the creation of crude yet functional 'bionic ears' containing an electronic device (Figure 10D) [67]. This device was fabricated by co-printing an alginate hydrogel matrix seeded with chondrocyte cells, and conducting silicone infused with silver particles, printed into a circular coil path to create an antenna. The seeded chondrocyte cells are then cultured into cartilage tissue, and the neo-cartilaginous tissue in contact with the printed electrode retains viability (Figure 10E). The interweaving of an electronic device into the organ allows the printed bionic ears to receive electromagnetic signals in a frequency range (up to $5 \mathrm{GHz}$ ) that is well beyond the normal perceptible range of human acoustic hearing $(20 \mathrm{~Hz}$ to $20 \mathrm{kHz}$ ) as shown in Figure 10F. 

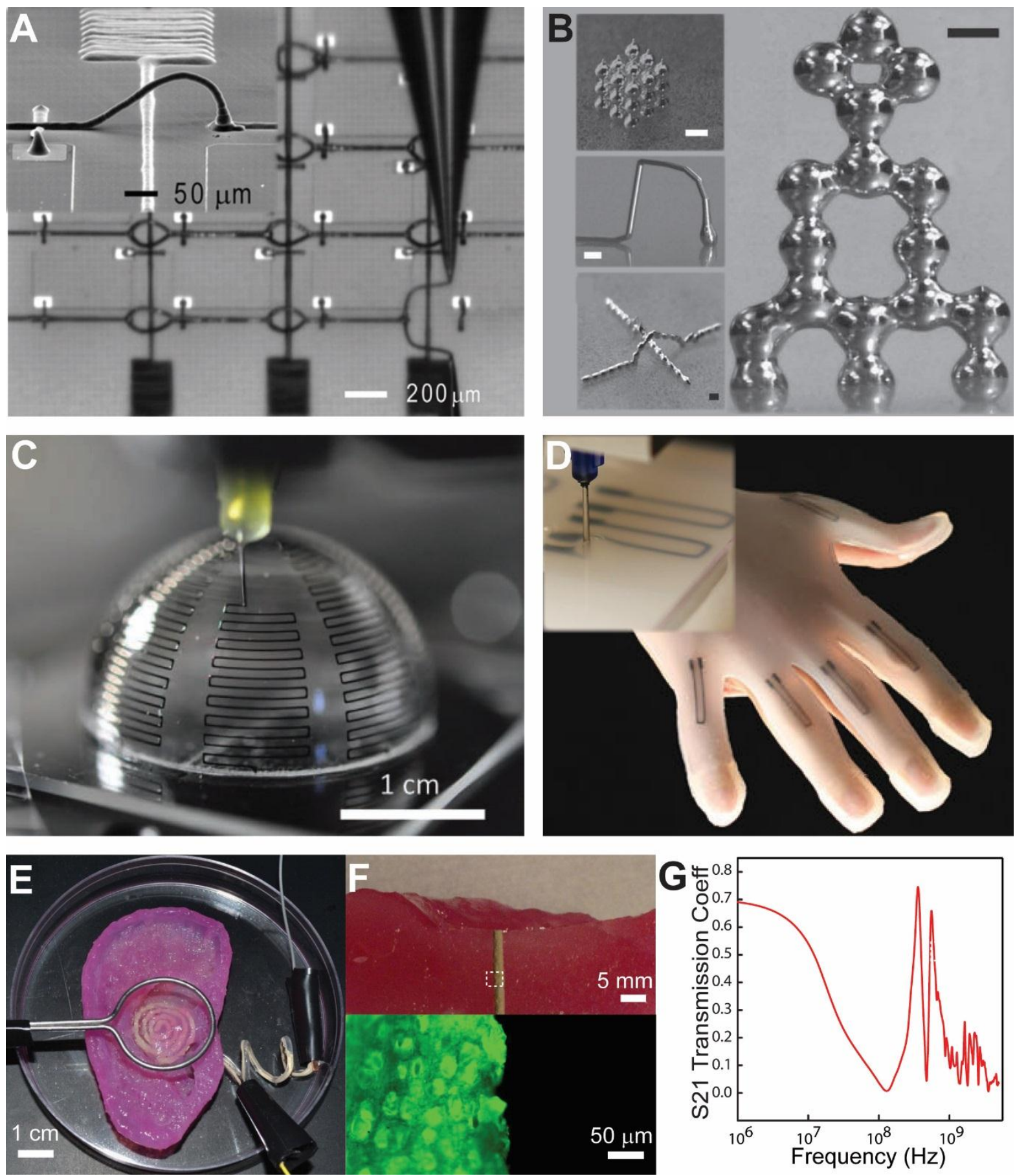

Figure 10. 3D printing of conducting electronic inks. (A) Omni-directional printing of a concentrated silver ink to form the interconnects of an LED chip array. The inset shows an interconnect arch printed over a junction [90]. (B) 3D printing of free-standing liquid metal into a cubic array of stacked droplets (top inset), a 3D metal arch (middle inset), an arch overpassing a 
printed wire (bottom inset), and a tower of liquid metal droplets. Scale bars are $500 \mu \mathrm{m}$ [70]. (C)

3D printing of a silver nanoparticle ink on a three dimensional surface to form an antenna [191]. (D) Embedded 3D printing of conducting carbon grease in an uncured elastomeric polymer (inset) enables the creation of stretchable strain sensors embedded within a glove [192]. (E) Co-printing of a conductor within a cell-laden biological scaffold to create a bionic ear. (F) Biocompatibility of the printed electronics within the biological construct. The fluorescent image (bottom) shows the viability of the neo-cartilaginous tissue in contact with the electrode (top) [67]. (G) Electromagnetic response of the 3D printed bionic ear. Plot shows the S21 transmission coefficient with frequency, demonstrating the capability of receiving signals over an expansive frequency range [67]. Reprinted with permission from Refs. [90], [70], [191], [192], [67], respectively. Copyright 2009 American Association for the Advancement of Science, 2011 John Wiley \& Sons, 2014 John Wiley \& Sons, 2013 American Chemical Society.

\section{D Printed Active Semiconducting Electronics}

Overcoming the planarity constraint of traditional microfabricated active electronic devices could enable the introduction of optoelectronic, sensing, and computational capabilities into non-flat, soft, flexible, and stretchable three dimensional constructs. For example, the incorporation of light emitting-diodes (LEDs) or sensors on contact lenses could provide components for on-eye wearable displays [193] or glucose sensors [194,195].

Indeed, developing the ability to 3D print active semiconducting materials is a critical and rapidly developing area which is expected to be a significant driver of 3D printing technologies going forward. For instance, Sun et al. demonstrated the 3D printing of a micro-battery via the coprinting of $\mathrm{Li}_{3} \mathrm{Ti}_{4} \mathrm{O}_{12}(\mathrm{LTO})$ and $\mathrm{LiFePO}_{4}(\mathrm{LFP})$ as cathode and anode, respectively (Figure 11A) 
[196]. The ability to stack the electrodes in a high aspect ratio form factor (up to 16 layers, Figure $11 \mathrm{~B})$ allows the 3D printed battery to achieve an exceptionally high energy density $\left(9.7 \mathrm{~J} \cdot \mathrm{cm}^{-2}\right.$ at a power density of $2.7 \mathrm{~mW} \cdot \mathrm{cm}^{-2}$ ). Further, the device exhibits a reasonable cycle life, as shown in Figure 11C, where only a small decay of areal capacity is observed after 30 charging cycles. Nevertheless, the fabrication process does include the deposition of gold on glass via electron beam evaporation. Further, a $600{ }^{\circ} \mathrm{C}$ heat treatment is required to remove organic additives and initiate the sintering process. Hence, further work is needed to 3D print such devices on threedimensional, temperature restrictive substrates.

The primary challenge in developing fully 3D printable active electronic devices such as diodes and transistors [197] lies in the complications associated with the integration of diverse classes of materials exhibiting disparate properties [47]. For instance, the 3D printing of LEDs requires the integration of a printable substrate, an emission layer, charge transport layers, a cathode, an anode and interconnects, all of which could consist of metal, semiconductor and polymeric materials, with varying surface energies, viscosities, and tribological and mechanical properties [61]. In general, the selection of inks needs to fulfill three major requirements. First, the materials have to be formulated into a 3D printable ink. Second, the ink materials have to retain their performance and function following extrusion from the 3D printer. For instance, in optoelectronic devices the bands must align properly [115]. Finally, the processing conditions and ink solvents have to be compatible with the other printed layers to minimize damage or degradation to already printed materials.

Recently, we have achieved this goal of fully 3D printing active semiconducting devices in the form of quantum dot light emitting diodes [61]. First, a transparent electrode consisting of a silver nanoparticle ring was printed, where the porous and hydrophilic nature of the ink allows for the 
formation of good electrical contact with a subsequently printed PEDOT:PSS layer. A charge transport layer (Poly TPD) and a nanoparticle semiconductor emissive layer (CdSe/ZnS QDs) are then 3D printed. The inks are formulated to form thin and uniform layers, as described previously. Finally, EGaIn liquid metal is printed to form a conformal top electrode. The printed device achieved a maximum brightness of $250 \mathrm{~cd} / \mathrm{m}^{2}$ at $5 \mathrm{~V}$ as shown in Figure $11 \mathrm{C}$, and the device also exhibited pure color emission from the QD emissive layer as shown in Figure 11D [115,121]. We anticipate that further optimization of the layer thicknesses and uniformities could further improve the device performances.

3D printing allows for a liberation of the device from the constraints of conventional microfabrication processes, such that the LEDs can be printed on non-planar and polymeric substrates. For instance, 3D scanning can be used to determine the precise topography of a nonplanar substrate. Then this information can be incorporated into a CAD program, such that the electronics can be conformally printed on the underlying 3D substrate. We validated this concept via the direct printing of a QD-LED on a 3D scanned contact lens (Figure 11E). This approach also allows us to build electronics up into the third dimension. Embedding these devices in three dimensions within an elastomeric structure enabled the creation of a $2 \times 2 \times 2$ array of multicolored LEDs embedded within a silicone cube, as shown in Figure 11F. This device fabrication approach of course is generalizable to other classes of devices, such as solar cells $[198,199]$ and transistors [197]. Further, the freeform fabrication of devices could also enable the creation of new classes of bionic devices with novel functionalities, such as soft optogenetic LED probes [14,200]. 

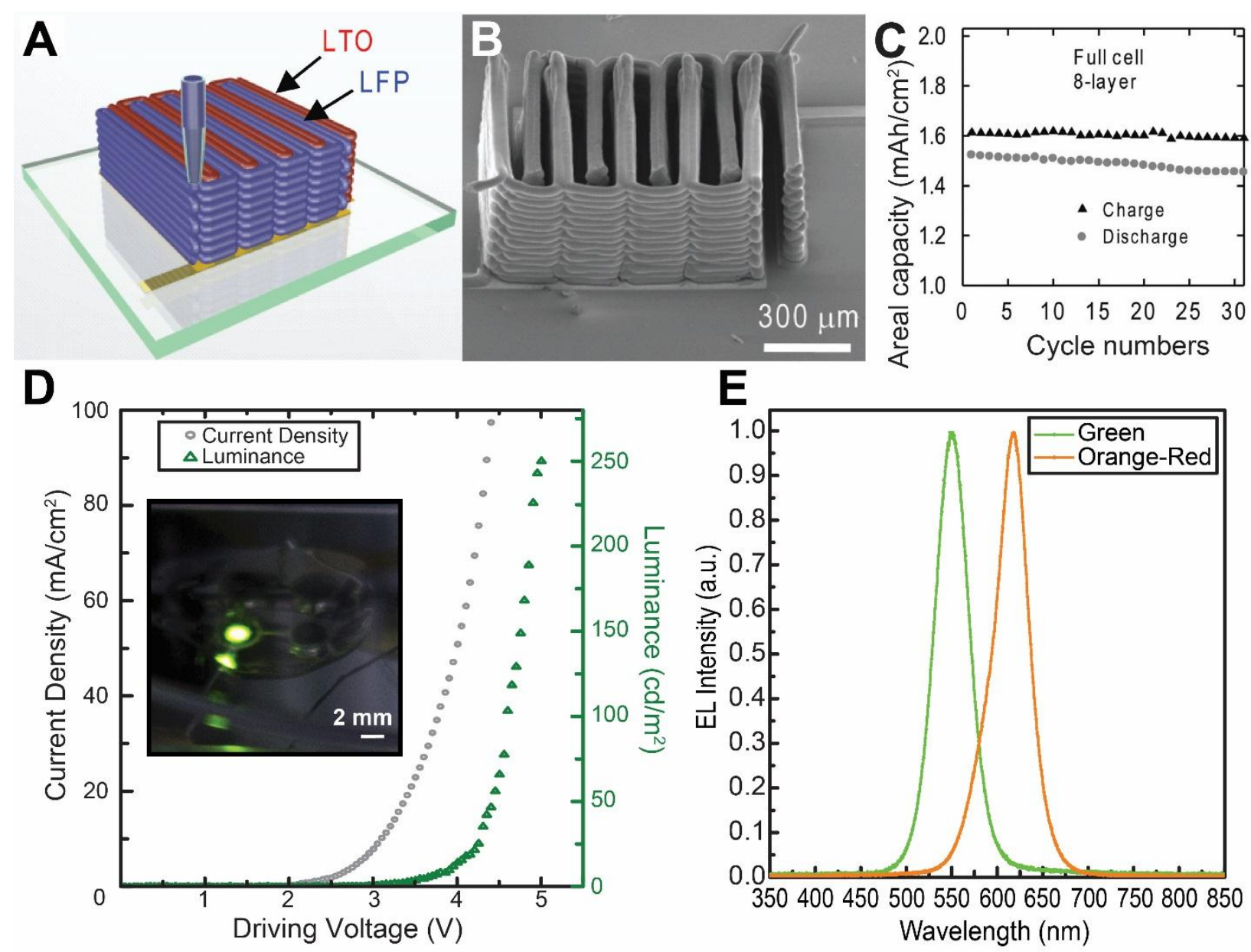

E
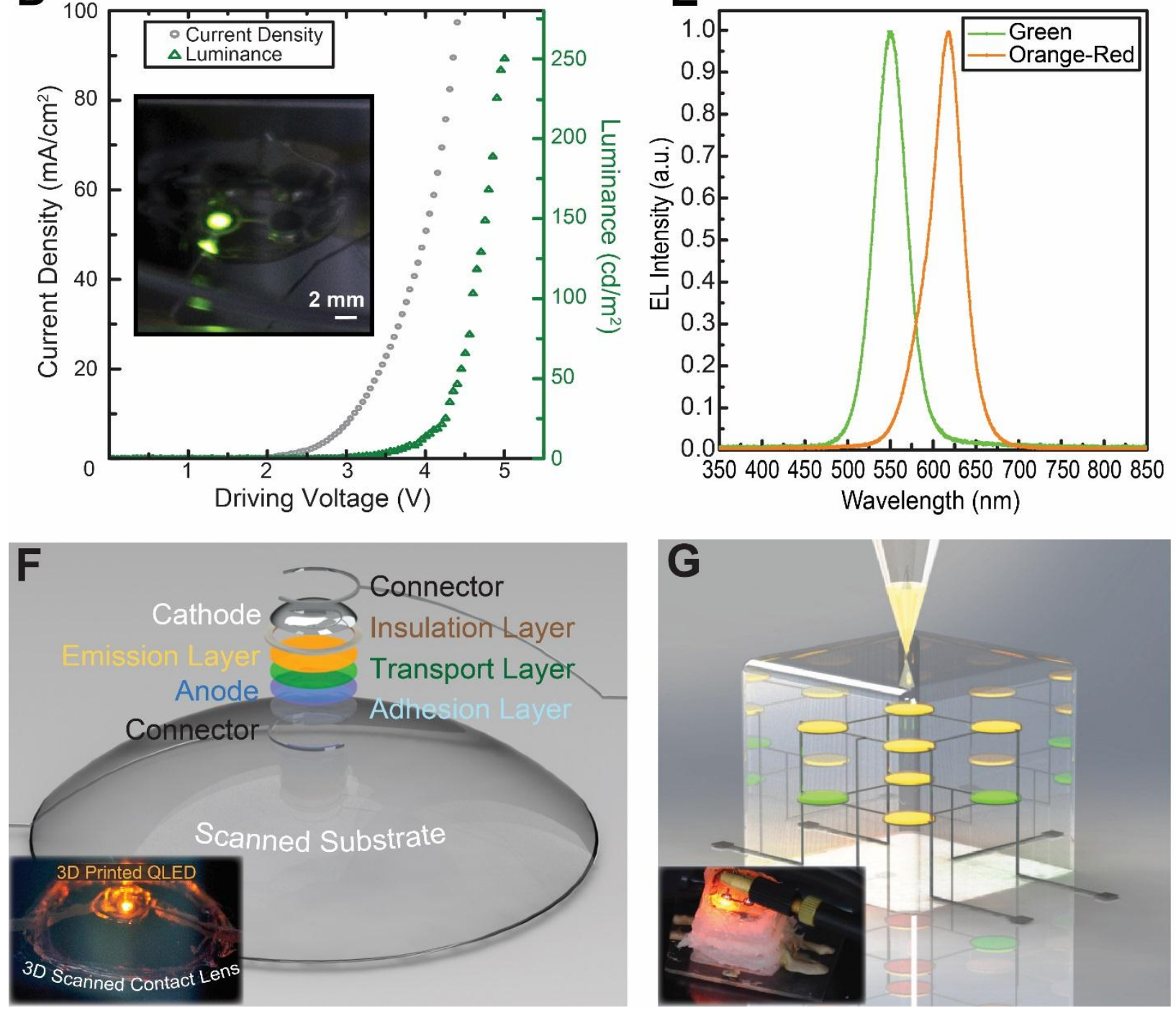

Figure 11. 3D printing of active electronics with semiconducting inks. (A) 3D Printing of $\mathrm{Li}_{3} \mathrm{Ti}_{4} \mathrm{O}_{12}(\mathrm{LTO})$ and $\mathrm{LiFePO}_{4}(\mathrm{LFP})$ inks to create a 3D interdigitated micro-battery architecture [196]. (B) SEM images of the printed 3D interdigitated micro-battery. (C) The cycle life of the 3D 
printed interdigitated battery. A good cycle life is achieved due to the low-strain topotactic reactions of LFP and LTO. (D) A 3D printed quantum dot light-emitting diode (QD-LED), where the inset shows the electroluminescence output from the QD-LED and the graph shows the current density vs. voltage and forward luminance output [61]. (E) Normalized electroluminescence spectra from both green and orange-red QD-LEDS, demonstrating color tunability and high color purity of the 3D printed QD-LEDS. (F) 3D printed QD-LED on a scanned curvilinear substrate, where the figure shows the CAD model and its components. The inset shows the electroluminescence output from the printed QD-LED on a 3D scanned contact lens [61]. (G) 3D printing of a $2 \times 2 \times 2$ multidimensional array of embedded QD-LEDs, where the inset shows the electroluminescence from a QD-LED in the 3D matrix [61]. Reprinted with permission from Refs. [196], [61], respectively. Copyright 2013 John Wiley \& Sons, 2014 American Chemical Society.

\section{Conclusion}

We have introduced a conceptually novel and comprehensive approach for the use of 3D printing as a versatile, multi-scale, multi-material tool that can address many of the fundamental challenges in the fabrication of bionic devices. 3D printing allows for the interweaving of the unique functionalities of nanomaterials with a variety of other materials, including soft and biological materials, to enable a seamless fabrication of three dimensional bionic devices. This is a multimaterial processing solution, which also achieves multiscale manufacturing - from nanoscale inks, to microscale features, to macroscale bionic devices. In this review, we have first described the unique properties of nanomaterials and their dispersion into functional inks. Second, we have reviewed the microscale printing of nanomaterials with 3D printing via convective self-assembly methods. We have also highlighted the versatility of 3D printing in creating hierarchical architectures, which can even include spatiotemporal gradients and stimuli-responsive capsules. 
Finally, we highlighted our ability to fabricate anatomically accurate macroscale bionic devices. The recent ability to extend these printing capabilities to active materials, such as semiconducting inks, is a critical development to impart complex functionalities into 3D printed devices that were previously simply passive constructs. Indeed, the ability to locally and directly print customized electronic devices into personalized 3D printed biomedical devices represents an exciting and extremely promising direction for future bioelectronics research. Overall, this blending of 3D printing, novel nanomaterial properties, and 'living' platforms may enable next-generation 3D printed bionic nanodevices.

\section{Acknowledgements:}

M.C.M. thanks the following agencies for their generous support of the studies from the authors' laboratory that contributed to this review: The Defense Advanced Research Projects Agency (No. D12AP00245), the Air Force Office of Scientific Research (No. FA9550-12-1-0368), the Intelligence Community (Award No. 2013-13070300004), and the National Institute of Biomedical Imaging and Bioengineering of the National Institutes of Health (Award No. 1DP2EB020537). The content is solely the responsibility of the authors and does not necessarily represent the official views of the National Institutes of Health. 


\section{References}

[1] M. Lavine, L. Roberts, O. Smith, Science 295 (2002) 995.

[2] W. Craelius, Science 295 (2002) 1018-1021.

[3] J. Clausen, Nature 457 (2009) 1080-1081.

[4] G. Vogel, Science 295 (2002) 1020.

[5] Dictionary.com Unabridged, Random House, Inc.

[6] R.F. Service, Science 340 (2013) 1162-1165.

[7] J.P. Giraldo, M.P. Landry, S.M. Faltermeier, T.P. McNicholas, N.M. Iverson, A.A.

Boghossian, N.F. Reuel, A.J. Hilmer, F. Sen, J.A. Brew, M.S. Strano, Nat. Mater. 13 (2014) 400408.

[8] E. Stavrinidou, R. Gabrielsson, E. Gomez, X. Crispin, O. Nilsson, D.T. Simon, M. Berggren, Sci. Adv. 1 (2015) e1501136.

[9] M. Haslam, A. Hernandez-Aguilar, V. Ling, S. Carvalho, I. de la Torre, A. DeStefano, A. Du, B. Hardy, J. Harris, L. Marchant, T. Matsuzawa, W. McGrew, J. Mercader, R. Mora, M.

Petraglia, H. Roche, E. Visalberghi, R. Warren, Nature 460 (2009) 339-344.

[10] E. Crubzy, P. Murail, L. Girard, J.-P. Bernadou, Nature 391 (1998) 29.

[11] D.H. Kim, N. Lu, R. Ma, Y.S. Kim, R.H. Kim, S. Wang, J. Wu, S.M. Won, H. Tao, A. Islam, K.J. Yu, T.I. Kim, R. Chowdhury, M. Ying, L. Xu, M. Li, H.J. Chung, H. Keum, M. McCormick, P. Liu, Y.W. Zhang, F.G. Omenetto, Y. Huang, T. Coleman, J.A. Rogers, Science 333 (2011) 838-843.

[12] B.C. Tee, A. Chortos, A. Berndt, A.K. Nguyen, A. Tom, A. McGuire, Z.C. Lin, K. Tien, W.G. Bae, H. Wang, P. Mei, H.H. Chou, B. Cui, K. Deisseroth, T.N. Ng, Z. Bao, Science 350 (2015) 313-316. 
[13] Y.M. Song, Y. Xie, V. Malyarchuk, J. Xiao, I. Jung, K.J. Choi, Z. Liu, H. Park, C. Lu, R.H.

Kim, R. Li, K.B. Crozier, Y. Huang, J.A. Rogers, Nature 497 (2013) 95-99.

[14] S.I. Park, D.S. Brenner, G. Shin, C.D. Morgan, B.A. Copits, H.U. Chung, M.Y. Pullen, K.N. Noh, S. Davidson, S.J. Oh, J. Yoon, K.I. Jang, V.K. Samineni, M. Norman, J.G. Grajales-Reyes, S.K. Vogt, S.S. Sundaram, K.M. Wilson, J.S. Ha, R. Xu, T. Pan, T.I. Kim, Y. Huang, M.C.

Montana, J.P. Golden, M.R. Bruchas, R.W. Gereau, J.A. Rogers, Nat. Biotechnol. 33 (2015) 1280-1286.

[15] M.A. McEvoy, N. Correll, Science 347 (2015) 1261689.

[16] D.-H. Kim, J.-H. Ahn, W.M. Choi, H.-S. Kim, T.-H. Kim, J. Song, Y.Y. Huang, Z. Liu, C. Lu, J.A. Rogers, Science 320 (2008) 507-511.

[17] B.S. Wilson, C.C. Finley, D.T. Lawson, R.D. Wolford, D.K. Eddington, W.M. Rabinowitz, Nature 352 (1991) 236-238.

[18] D.R. Moore, R.V. Shannon, Nature Neuro. 12 (2009) 686-691.

[19] M.F. Eilenberg, H. Geyer, H. Herr, IEEE Trans. Neural Syst. Rehabil. Eng. 18 (2010) 164173.

[20] L. da Cruz, B.F. Coley, J. Dorn, F. Merlini, E. Filley, P. Christopher, F.K. Chen, V.

Wuyyuru, J. Sahel, P. Stanga, M. Humayun, R.J. Greenberg, G. Dagnelie, Br. J. Ophthalmol. 97 (2013) 632-636.

[21] E. Zrenner, Sci. Transl. Med 5 (2013) 210ps216.

[22] I.R. Minev, P. Musienko, A. Hirsch, Q. Barraud, N. Wenger, E.M. Moraud, J. Gandar, M. Capogrosso, T. Milekovic, L. Asboth, R.F. Torres, N. Vachicouras, Q. Liu, N. Pavlova, S. Duis, A. Larmagnac, J. Voros, S. Micera, Z. Suo, G. Courtine, S.P. Lacour, Science 347 (2015) 159163. 
[23] M.L. Hammock, A. Chortos, B.C.K. Tee, J.B.H. Tok, Z. Bao, Adv. Mater. 25 (2013) 59976038.

[24] T. Someya, T. Sekitani, S. Iba, Y. Kato, H. Kawaguchi, T. Sakurai, Proc. Nat. Acad. Sci. U.S.A. 101 (2004) 9966-9970.

[25] D.J. Lipomi, M. Vosgueritchian, B.C.K. Tee, S.L. Hellstrom, J.A. Lee, C.H. Fox, Z. Bao, Nat. Nanotechnol. 6 (2011) 788-792.

[26] D. Farina, O. Aszmann, Sci. Transl. Med 6 (2014) 257ps212.

[27] J.M. Anderson, A.K. McNally, Semin Immunopathol. 33 (2011) 221-233.

[28] L. Zhang, Z. Cao, T. Bai, L. Carr, J.-R. Ella-Menye, C. Irvin, B.D. Ratner, S. Jiang, Nat. Biotechnol. 31 (2013) 553-556.

[29] O. Veiseh, J.C. Doloff, M. Ma, A.J. Vegas, H.H. Tam, A.R. Bader, J. Li, E. Langan, J. Wyckoff, W.S. Loo, S. Jhunjhunwala, A. Chiu, S. Siebert, K. Tang, J. Hollister-Lock, S. ArestaDasilva, M. Bochenek, J. Mendoza-Elias, Y. Wang, M. Qi, D.M. Lavin, M. Chen, N. Dholakia, R. Thakrar, I. Lacik, G.C. Weir, J. Oberholzer, D.L. Greiner, R. Langer, D.G. Anderson, Nat. Mater. 14 (2015) 643-651.

[30] M.F. Ashby, Materials Selection in Mechanical Design, 1st ed., Pergamon Press, Oxford; New York, 1992.

[31] P.G. Agache, C. Monneur, J.L. Leveque, J. De Rigal, Arch. Dermatol. Res. 269 (1980) 221232.

[32] J.A. Rogers, T. Someya, Y. Huang, Science 327 (2010) 1603-1607.

[33] M.A. Nicolelis, D. Dimitrov, J.M. Carmena, R. Crist, G. Lehew, J.D. Kralik, S.P. Wise, Proc. Nat. Acad. Sci. U.S.A. 100 (2003) 11041-11046. 
[34] S. Bai, S. Debnath, K. Gibson, B. Schlicht, L. Bayne, M. Zagnoni, R.V. Ulijn, Small 10 (2014) 285-293.

[35] P. Fratzl, R. Weinkamer, Prog. Mater Sci. 52 (2007) 1263-1334.

[36] R.H. Kim, D.H. Kim, J. Xiao, B.H. Kim, S.I. Park, B. Panilaitis, R. Ghaffari, J. Yao, M. Li, Z. Liu, V. Malyarchuk, D.G. Kim, A.P. Le, R.G. Nuzzo, D.L. Kaplan, F.G. Omenetto, Y. Huang, Z. Kang, J.A. Rogers, Nat. Mater. 9 (2010) 929-937.

[37] J.A. Rogers, M.G. Lagally, R.G. Nuzzo, Nature 477 (2011) 45-53.

[38] M.C. McAlpine, H. Ahmad, D. Wang, J.R. Heath, Nat. Mater. 6 (2007) 379-384.

[39] C. Dagdeviren, B.D. Yang, Y. Su, P.L. Tran, P. Joe, E. Anderson, J. Xia, V. Doraiswamy, B. Dehdashti, X. Feng, B. Lu, R. Poston, Z. Khalpey, R. Ghaffari, Y. Huang, M.J. Slepian, J.A. Rogers, Proc. Nat. Acad. Sci. U.S.A. 111 (2014) 1927-1932.

[40] D.-H. Kim, J. Viventi, J.J. Amsden, J. Xiao, L. Vigeland, Y.-S. Kim, J.A. Blanco, B.

Panilaitis, E.S. Frechette, D. Contreras, D.L. Kaplan, F.G. Omenetto, Y. Huang, K.-C. Hwang, M.R. Zakin, B. Litt, J.A. Rogers, Nat. Mater. 9 (2010) 511-517.

[41] D.-H. Kim, N. Lu, R. Ghaffari, J.A. Rogers, NPG Asia Mater 4 (2012) e15.

[42] S.A. Morin, Y. Shevchenko, J. Lessing, S.W. Kwok, R.F. Shepherd, A.A. Stokes, G.M. Whitesides, Adv. Mater. 26 (2014) 5991-5999.

[43] C.W. Hull, UVP, Inc., US, March 11, 1986.

[44] R.A. Jarvis, IEEE Trans. Pattern Anal. Mach. Intell. PAMI-5 (1983) 122-139.

[45] C. Rocchini, P. Cignoni, C. Montani, P. Pingi, R. Scopigno, Comput. Graph. Forum 20 (2001) 299-308.

[46] J. Salvi, J. Pagès, J. Batlle, Pattern Recognit. 37 (2004) 827-849.

[47] J.R. Raney, J.A. Lewis, MRS Bull. 40 (2015) 943-950. 
[48] X. Zhang, X. Jiang, C. Sun, Sens. Actuat. A - Phys. 77 (1999) 149-156.

[49] C. Sun, N. Fang, D. Wu, X. Zhang, Sens. Actuat. A - Phys. 121 (2005) 113-120.

[50] B.H. Cumpston, S.P. Ananthavel, S. Barlow, D.L. Dyer, J.E. Ehrlich, L.L. Erskine, A.A.

Heikal, S.M. Kuebler, I.-Y.S. Lee, D. McCord-Maughon, Nature 398 (1999) 51-54.

[51] P. Calvert, Chem. Mater. 13 (2001) 3299-3305.

[52] M.S. Onses, E. Sutanto, P.M. Ferreira, A.G. Alleyne, J.A. Rogers, Small 11 (2015) 42374266.

[53] H. Lipson, M. Kurman, Fabricated : The New World of 3D Printing, John Wiley and Sons, Inc., Indianapolis,IN, 2013.

[54] E. Malone, H. Lipson, Rapid Prototyping J. 13 (2007) 245-255.

[55] S.V. Murphy, A. Atala, Nat. Biotechnol. 32 (2014) 773-785.

[56] J.A. Lewis, J.E. Smay, J. Stuecker, J. Cesarano, J. Am. Ceram. Soc. 89 (2006) 3599-3609.

[57] J.A. Lewis, Adv. Funct. Mater. 16 (2006) 2193-2204.

[58] J.M. Pearce, Science 337 (2012) 1303-1304.

[59] T.J. Ober, D. Foresti, J.A. Lewis, Proc. Nat. Acad. Sci. U.S.A. 112 (2015) 12293-12298.

[60] J.O. Hardin, T.J. Ober, A.D. Valentine, J.A. Lewis, Adv. Mater. 27 (2015) 3279-3284.

[61] Y.L. Kong, I.A. Tamargo, H. Kim, B.N. Johnson, M.K. Gupta, T.W. Koh, H.A. Chin, D.A.

Steingart, B.P. Rand, M.C. McAlpine, Nano Lett. 14 (2014) 7017-7023.

[62] M.K. Gupta, F. Meng, B.N. Johnson, Y.L. Kong, L. Tian, Y.-W. Yeh, N. Masters, S.

Singamaneni, M.C. McAlpine, Nano Lett. 15 (2015) 5321-5329.

[63] B.G. Compton, J.A. Lewis, Adv. Mater. 26 (2014) 5930-5935. 
[64] B.N. Johnson, K.Z. Lancaster, G. Zhen, J. He, M.K. Gupta, Y.L. Kong, E.A. Engel, K.D.

Krick, A. Ju, F. Meng, L.W. Enquist, X. Jia, M.C. McAlpine, Adv. Funct. Mater. 25 (2015) 6205-6217.

[65] B.N. Johnson, K.Z. Lancaster, I.B. Hogue, F. Meng, Y.L. Kong, L.W. Enquist, M.C. McAlpine, Lab Chip (2015).

[66] J.A. Lewis, Tissue Eng. Pt. A 21 (2015) S22-S22.

[67] M.S. Mannoor, Z. Jiang, T. James, Y.L. Kong, K.A. Malatesta, W.O. Soboyejo, N. Verma, D.H. Gracias, M.C. McAlpine, Nano Lett. 13 (2013) 2634-2639.

[68] J.A. Lewis, Curr. Opin. Solid State Mater. Sci. 6 (2002) 245-250.

[69] S.L. Morissette, J.A. Lewis, P.G. Clem, J. Cesarano, D.B. Dimos, J. Am. Ceram. Soc. 84 (2001) 2462-2468.

[70] C. Ladd, J.H. So, J. Muth, M.D. Dickey, Adv. Mater. 25 (2013) 5081-5085.

[71] A. Frutiger, J.T. Muth, D.M. Vogt, Y. Menguc, A. Campo, A.D. Valentine, C.J. Walsh, J.A. Lewis, Adv. Mater. 27 (2015) 2440-2446.

[72] J.N. Hanson Shepherd, S.T. Parker, R.F. Shepherd, M.U. Gillette, J.A. Lewis, R.G. Nuzzo, Adv. Funct. Mater. 21 (2011) 47-54.

[73] R.A. Barry, R.F. Shepherd, J.N. Hanson, R.G. Nuzzo, P. Wiltzius, J.A. Lewis, Adv. Mater. 21 (2009) 2407-2410.

[74] M.J. Webber, E.A. Appel, E.W. Meijer, R. Langer, Nat. Mater. 15 (2015) 13-26.

[75] R.P. Feynman, Eng. Sci. 23 (1960) 22-36.

[76] Y. Huang, X. Duan, Q. Wei, C.M. Lieber, Science 291 (2001) 630-633.

[77] X. Chen, S.S. Mao, Chem. Rev. 107 (2007) 2891-2959. 
[78] B. Dabbousi, J. Rodriguez-Viejo, F.V. Mikulec, J. Heine, H. Mattoussi, R. Ober, K. Jensen, M. Bawendi, J. Phys. Chem. B 101 (1997) 9463-9475.

[79] A.S. Edelstein, R. Cammaratra, Nanomaterials: synthesis, properties and applications, CRC Press 1998.

[80] C.B. Murray, S. Sun, W. Gaschler, H. Doyle, T.A. Betley, C.R. Kagan, IBM J. Res. Dev. 45 (2001) 47-56.

[81] C.N.R. Rao, A. Müller, A.K. Cheetham, The chemistry of nanomaterials: synthesis, properties and applications, John Wiley \& Sons2006.

[82] J.N. Israelachvili, Intermolecular and surface forces, 3rd ed., Academic Press, Burlington, MA, 2011.

[83] Y. Min, M. Akbulut, K. Kristiansen, Y. Golan, J. Israelachvili, Nat. Mater. 7 (2008) 527538.

[84] S.P. Koenig, N.G. Boddeti, M.L. Dunn, J.S. Bunch, Nat. Nanotechnol. 6 (2011) 543-546.

[85] E. Roduner, Chem. Soc. Rev. 35 (2006) 583-592.

[86] H. Goesmann, C. Feldmann, Angew. Chem. Int. Ed. 49 (2010) 1362-1395.

[87] P. Couchman, W. Jesser, Nature 269 (1977) 481 - 483.

[88] S.L. Lai, J.Y. Guo, V.V. Petrova, G. Ramanath, L.H. Allen, Phys. Rev. Lett. 77 (1996) 99102.

[89] W. Thomson, Proc. R. Soc. Edinburgh 7 (1872) 63-68.

[90] B.Y. Ahn, E.B. Duoss, M.J. Motala, X. Guo, S.I. Park, Y. Xiong, J. Yoon, R.G. Nuzzo, J.A. Rogers, J.A. Lewis, Science 323 (2009) 1590-1593.

[91] M. Faraday, Phil. Trans. R. Soc. Lond. 147 (1857) 145-181.

[92] A.P. Alivisatos, Science 271 (1996) 933-937. 
[93] A. Eychmüller, J. Phys. Chem. B 104 (2000) 6514-6528.

[94] T. Witten, P. Pincus, Macromolecules 19 (1986) 2509-2513.

[95] D. Li, M.B. Muller, S. Gilje, R.B. Kaner, G.G. Wallace, Nat. Nanotechnol. 3 (2008) 101105.

[96] A. Kamyshny, S. Magdassi, Small 10 (2014) 3515-3535.

[97] Y. Sun, Y. Xia, Science 298 (2002) 2176-2179.

[98] D.S. Hecht, L. Hu, G. Irvin, Adv. Mater. 23 (2011) 1482-1513.

[99] K. Chopra, S. Major, D. Pandya, Thin Solid Films 102 (1983) 1-46.

[100] F. Bonaccorso, Z. Sun, T. Hasan, A. Ferrari, Nat. Photon. 4 (2010) 611-622.

[101] G. Eda, G. Fanchini, M. Chhowalla, Nat. Nanotechnol. 3 (2008) 270-274.

[102] Y. Li, Y. Wu, B.S. Ong, J. Am. Chem. Soc. 127 (2005) 3266-3267.

[103] M. Brust, M. Walker, D. Bethell, D.J. Schiffrin, R. Whyman, J. Chem. Soc., Chem. Commun. (1994) 801-802.

[104] M. Brust, J. Fink, D. Bethell, D. Schiffrin, C. Kiely, J. Chem. Soc., Chem. Commun. (1995) 1655-1656.

[105] T. Campbell, R.K. Kalia, A. Nakano, P. Vashishta, S. Ogata, S. Rodgers, Phys. Rev. Lett. 82 (1999) 4866.

[106] K.S. Novoselov, D. Jiang, F. Schedin, T.J. Booth, V.V. Khotkevich, S.V. Morozov, A.K. Geim, Proc. Nat. Acad. Sci. U.S.A. 102 (2005) 10451-10453.

[107] A.K. Geim, K.S. Novoselov, Nat. Mater. 6 (2007) 183-191.

[108] A.K. Geim, Science 324 (2009) 1530-1534.

[109] K.I. Bolotin, K. Sikes, Z. Jiang, M. Klima, G. Fudenberg, J. Hone, P. Kim, H. Stormer, Solid State Commun. 146 (2008) 351-355. 
[110] A.H. Castro Neto, F. Guinea, N.M.R. Peres, K.S. Novoselov, A.K. Geim, Rev. Mod. Phys. 81 (2009) 109-162.

[111] S. Wang, P.K. Ang, Z. Wang, A.L. Tang, J.T. Thong, K.P. Loh, Nano Lett. 10 (2010) 9298.

[112] K.-Y. Chun, Y. Oh, J. Rho, J.-H. Ahn, Y.-J. Kim, H.R. Choi, S. Baik, Nat. Nanotechnol. 5 (2010) 853-857.

[113] P.O. Anikeeva, J.E. Halpert, M.G. Bawendi, V. Bulović, Nano Lett. 7 (2007) 2196-2200.

[114] O. Chen, J. Zhao, V.P. Chauhan, J. Cui, C. Wong, D.K. Harris, H. Wei, H.S. Han, D.

Fukumura, R.K. Jain, M.G. Bawendi, Nat. Mater. 12 (2013) 445-451.

[115] Y. Shirasaki, G.J. Supran, M.G. Bawendi, V. Bulovic, Nat. Photon. 7 (2013) 13-23.

[116] L.A. Austin, B. Kang, M.A. El-Sayed, Nano Today 10 (2015) 542-558.

[117] I. Gur, N.A. Fromer, M.L. Geier, A.P. Alivisatos, Science 310 (2005) 462-465.

[118] S.A. McDonald, G. Konstantatos, S. Zhang, P.W. Cyr, E.J. Klem, L. Levina, E.H. Sargent, Nat. Mater. 4 (2005) 138-142.

[119] M. Graetzel, R.A. Janssen, D.B. Mitzi, E.H. Sargent, Nature 488 (2012) 304-312.

[120] J. Yang, M.K. Choi, D.H. Kim, T. Hyeon, Adv. Mater. (2015).

[121] L. Qian, Y. Zheng, J. Xue, P.H. Holloway, Nat. Photon. 5 (2011) 543-548.

[122] G.J. Supran, Y. Shirasaki, K.W. Song, J.-M. Caruge, P.T. Kazlas, S. Coe-Sullivan, T.L. Andrew, M.G. Bawendi, V. Bulović, MRS Bull. 38 (2013) 703-711.

[123] R.D. Deegan, O. Bakajin, T.F. Dupont, G. Huber, S.R. Nagel, T.A. Witten, Nature 389 (1997) 827-829.

[124] R.D. Deegan, Phys. Rev. E. 61 (2000) 475-485.

[125] L. Shmuylovich, A.Q. Shen, H.A. Stone, Langmuir 18 (2002) 3441-3445. 
[126] K.M. Ryan, A. Mastroianni, K.A. Stancil, H. Liu, A.P. Alivisatos, Nano Lett. 6 (2006) 1479-1482.

[127] S.S.H. Tsai, J.S. Wexler, J. Wan, H.A. Stone, Appl. Phys. Lett. 99 (2011) 153509.

[128] J. Park, J. Moon, Langmuir 22 (2006) 3506-3513.

[129] D.J. Harris, J.A. Lewis, Langmuir 24 (2008) 3681-3685.

[130] H. Hu, R.G. Larson, J. Phys. Chem. B 110 (2006) 7090-7094.

[131] M. Majumder, C.S. Rendall, J.A. Eukel, J.Y.L. Wang, N. Behabtu, C.L. Pint, T.-Y. Liu, A.W. Orbaek, F. Mirri, J. Nam, A.R. Barron, R.H. Hauge, H.K. Schmidt, M. Pasquali, J. Phys. Chem. B 116 (2012) 6536-6542.

[132] T. Still, P.J. Yunker, A.G. Yodh, Langmuir 28 (2012) 4984-4988.

[133] L. Cui, J. Zhang, X. Zhang, L. Huang, Z. Wang, Y. Li, H. Gao, S. Zhu, T. Wang, B. Yang, ACS Appl. Mater. Interfaces 4 (2012) 2775-2780.

[134] D. Soltman, V. Subramanian, Langmuir 24 (2008) 2224-2231.

[135] P.J. Yunker, T. Still, M.A. Lohr, A.G. Yodh, Nature 476 (2011) 308-311.

[136] T.P. Bigioni, X.-M. Lin, T.T. Nguyen, E.I. Corwin, T.A. Witten, H.M. Jaeger, Nat. Mater. 5 (2006) 265-270.

[137] A. Shimoni, S. Azoubel, S. Magdassi, Nanoscale 6 (2014) 11084-11089.

[138] J. Xu, J. Xia, Z. Lin, Angew. Chem. Int. Ed. 46 (2007) 1860-1863.

[139] S. Watanabe, K. Inukai, S. Mizuta, M.T. Miyahara, Langmuir 25 (2009) 7287-7295.

[140] M. Abkarian, J. Nunes, H.A. Stone, J. Am. Chem. Soc. 126 (2004) 5978-5979.

[141] Y.L. Kong, F. Boulogne, H. Kim, J. Nunes, J. Feng, H.A. Stone, Langmuir 31 (2015) 12560-12566. 
[142] H.S. Kim, C.H. Lee, P.K. Sudeep, T. Emrick, A.J. Crosby, Adv. Mater. 22 (2010) 46004604.

[143] L. Wang, J. Lau, E.L. Thomas, M.C. Boyce, Adv. Mater. 23 (2011) 1524-1529.

[144] L.S. Dimas, G.H. Bratzel, I. Eylon, M.J. Buehler, Adv. Funct. Mater. 23 (2013) 46294638.

[145] T.A. Schaedler, A.J. Jacobsen, A. Torrents, A.E. Sorensen, J. Lian, J.R. Greer, L. Valdevit, W.B. Carter, Science 334 (2011) 962-965.

[146] L.R. Meza, S. Das, J.R. Greer, Science 345 (2014) 1322-1326.

[147] G.M. Gratson, F. García-Santamaría, V. Lousse, M. Xu, S. Fan, J.A. Lewis, P.V. Braun, Adv. Mater. 18 (2006) 461-465.

[148] A. Clausen, F.W. Wang, J.S. Jensen, O. Sigmund, J.A. Lewis, Adv. Mater. 27 (2015) 5523-5527.

[149] S. Shan, S.H. Kang, J.R. Raney, P. Wang, L. Fang, F. Candido, J.A. Lewis, K. Bertoldi, Adv. Mater. 27 (2015) 4296-4301.

[150] C.J. Hansen, R. Saksena, D.B. Kolesky, J.J. Vericella, S.J. Kranz, G.P. Muldowney, K.T. Christensen, J.A. Lewis, Adv. Mater. 25 (2013) 96-102.

[151] M. Biondi, F. Ungaro, F. Quaglia, P.A. Netti, Adv. Drug Deliv. Rev. 60 (2008) 229-242.

[152] L.G. Griffith, M.A. Swartz, Nat. Rev. Mol. Cell. Biol. 7 (2006) 211-224.

[153] M.P. Lutolf, P.M. Gilbert, H.M. Blau, Nature 462 (2009) 433-441.

[154] F.M. Chen, M. Zhang, Z.F. Wu, Biomaterials 31 (2010) 6279-6308.

[155] T.M. Keenan, A. Folch, Lab Chip 8 (2008) 34-57.

[156] M.P. Lutolf, H.M. Blau, Adv. Mater. 21 (2009) 3255-3268. 
[157] U.A. Gurkan, Y. Fan, F. Xu, B. Erkmen, E.S. Urkac, G. Parlakgul, J. Bernstein, W. Xing, E.S. Boyden, U. Demirci, Adv. Mater. 25 (2013) 1192-1198.

[158] S. Ilkhanizadeh, A.I. Teixeira, O. Hermanson, Biomaterials 28 (2007) 3936-3943.

[159] E.D. Ker, B. Chu, J.A. Phillippi, B. Gharaibeh, J. Huard, L.E. Weiss, P.G. Campbell, Biomaterials 32 (2011) 3413-3422.

[160] E.D. Ker, A.S. Nain, L.E. Weiss, J. Wang, J. Suhan, C.H. Amon, P.G. Campbell, Biomaterials 32 (2011) 8097-8107.

[161] E.D. Miller, K. Li, T. Kanade, L.E. Weiss, L.M. Walker, P.G. Campbell, Biomaterials 32 (2011) 2775-2785.

[162] E.D. Miller, J.A. Phillippi, G.W. Fisher, P.G. Campbell, L.M. Walker, L.E. Weiss, Comb. Chem. High Throughput Screen 12 (2009) 604-618.

[163] H. Zhang, J.N.H. Shepherd, R.G. Nuzzo, Soft Matter 6 (2010) 2238-2245.

[164] M.P. Chien, A.M. Rush, M.P. Thompson, N.C. Gianneschi, Angew. Chem. Int. Ed. 49 (2010) 5076-5080.

[165] M.H. Lee, K.C. Hribar, T. Brugarolas, N.P. Kamat, J.A. Burdick, D. Lee, Adv. Funct. Mater. 22 (2012) 131-138.

[166] F. Mohamed, C.F. van der Walle, J. Pharm. Sci. 97 (2008) 71-87.

[167] R.K. Shah, H.C. Shum, A.C. Rowat, D. Lee, J.J. Agresti, A.S. Utada, L.-Y. Chu, J.-W.

Kim, A. Fernandez-Nieves, C.J. Martinez, Mater. Today 11 (2008) 18-27.

[168] A.S. Utada, E. Lorenceau, D.R. Link, P.D. Kaplan, H.A. Stone, D.A. Weitz, Science 308 (2005) 537-541.

[169] S. Xu, Z. Nie, M. Seo, P. Lewis, E. Kumacheva, H.A. Stone, P. Garstecki, D.B. Weibel, I. Gitlin, G.M. Whitesides, Angew. Chem. Int. Ed. 117 (2005) 734-738. 
[170] R.K. Shah, J.W. Kim, D.A. Weitz, Langmuir 26 (2010) 1561-1565.

[171] L. Tian, N. Gandra, S. Singamaneni, ACS Nano 7 (2013) 4252-4260.

[172] B.P. Timko, M. Arruebo, S.A. Shankarappa, J.B. McAlvin, O.S. Okonkwo, B. Mizrahi, C.F. Stefanescu, L. Gomez, J. Zhu, A. Zhu, J. Santamaria, R. Langer, D.S. Kohane, Proc. Nat. Acad. Sci. U.S.A. 111 (2014) 1349-1354.

[173] Y. Wang, J.D. Byrne, M.E. Napier, J.M. DeSimone, Adv. Drug Deliv. Rev. 64 (2012) 1021-1030.

[174] Y. Luo, M.S. Shoichet, Nat. Mater. 3 (2004) 249-253.

[175] N.W. Choi, M. Cabodi, B. Held, J.P. Gleghorn, L.J. Bonassar, A.D. Stroock, Nat. Mater. 6 (2007) 908-915.

[176] S. Kobel, M.P. Lutolf, Curr. Opin. Biotechnol. 22 (2011) 690-697.

[177] T. Bhattacharjee, S.M. Zehnder, K.G. Rowe, S. Jain, R.M. Nixon, W.G. Sawyer, T.E. Angelini, Sci. Adv. 1 (2015) e1500655.

[178] D.B. Kolesky, R.L. Truby, A.S. Gladman, T.A. Busbee, K.A. Homan, J.A. Lewis, Adv. Mater. 26 (2014) 3124-3130.

[179] J.S. Miller, K.R. Stevens, M.T. Yang, B.M. Baker, D.H. Nguyen, D.M. Cohen, E. Toro, A.A. Chen, P.A. Galie, X. Yu, R. Chaturvedi, S.N. Bhatia, C.S. Chen, Nat. Mater. 11 (2012) 768-774.

[180] D. Ibrahim, T.L. Broilo, C. Heitz, M.G. de Oliveira, H.W. de Oliveira, S.M.W. Nobre, J.H.G. dos Santos Filho, D.N. Silva, J. Craniomaxillofac. Surg. 37 (2009) 167-173.

[181] B. Duan, E. Kapetanovic, L.A. Hockaday, J.T. Butcher, Acta Biomater. 10 (2014) 18361846. 
[182] J.P. Temple, D.L. Hutton, B.P. Hung, P.Y. Huri, C.A. Cook, R. Kondragunta, X. Jia, W.L. Grayson, Journal of Biomedical Materials Research Part A 102 (2014) 4317-4325.

[183] L. Wen, J.C. Weaver, G.V. Lauder, J. Exp. Biol. 217 (2014) 1656-1666.

[184] V. Mironov, T. Boland, T. Trusk, G. Forgacs, R.R. Markwald, Trends Biotechnol. 21 (2003) 157-161.

[185] H. Seitz, W. Rieder, S. Irsen, B. Leukers, C. Tille, J Biomed Mater Res B Appl Biomater 74 (2005) 782-788.

[186] Z. Qin, B.G. Compton, J.A. Lewis, M.J. Buehler, Nat. Commun. 6 (2015) 7038.

[187] B.Y. Ahn, D.J. Lorang, J.A. Lewis, Nanoscale 3 (2011) 2700-2702.

[188] R.D. Piner, J. Zhu, F. Xu, S. Hong, C.A. Mirkin, Science 283 (1999) 661-663.

[189] R.C. Chiechi, E.A. Weiss, M.D. Dickey, G.M. Whitesides, Angew. Chem. Int. Ed. 47 (2008) 142-144.

[190] J.W. Boley, E.L. White, G.T.C. Chiu, R.K. Kramer, Adv. Funct. Mater. 24 (2014) 35013507.

[191] J.J. Adams, E.B. Duoss, T.F. Malkowski, M.J. Motala, B.Y. Ahn, R.G. Nuzzo, J.T. Bernhard, J.A. Lewis, Adv. Mater. 23 (2011) 1335-1340.

[192] J.T. Muth, D.M. Vogt, R.L. Truby, Y. Menguc, D.B. Kolesky, R.J. Wood, J.A. Lewis, Adv. Mater. 26 (2014) 6307-6312.

[193] M.S. Lee, K. Lee, S.Y. Kim, H. Lee, J. Park, K.H. Choi, H.K. Kim, D.G. Kim, D.Y. Lee, S. Nam, J.U. Park, Nano Lett. 13 (2013) 2814-2821.

[194] H. Yao, A.J. Shum, M. Cowan, I. Lahdesmaki, B.A. Parviz, Biosens. Bioelectron. 26 (2011) 3290-3296.

[195] A.J. Bandodkar, J. Wang, Trends Biotechnol. 32 (2014) 363-371. 
[196] K. Sun, T.S. Wei, B.Y. Ahn, J.Y. Seo, S.J. Dillon, J.A. Lewis, Adv. Mater. 25 (2013) 4539-4543.

[197] A.D. Franklin, Science 349 (2015) aab2750.

[198] E.H. Sargent, Adv. Mater. 20 (2008) 3958-3964.

[199] A.G. Pattantyus-Abraham, I.J. Kramer, A.R. Barkhouse, X. Wang, G. Konstantatos, R.

Debnath, L. Levina, I. Raabe, M.K. Nazeeruddin, M. Gratzel, E.H. Sargent, ACS Nano 4 (2010) 3374-3380.

[200] C. Goßler, C. Bierbrauer, R. Moser, M. Kunzer, K. Holc, W. Pletschen, K. Köhler, J.

Wagner, M. Schwaerzle, P. Ruther, O. Paul, J. Neef, D. Keppeler, G. Hoch, T. Moser, U.T.

Schwarz, J. Phys. D: Appl. Phys. 47 (2014) 205401.

Graphical Abstract

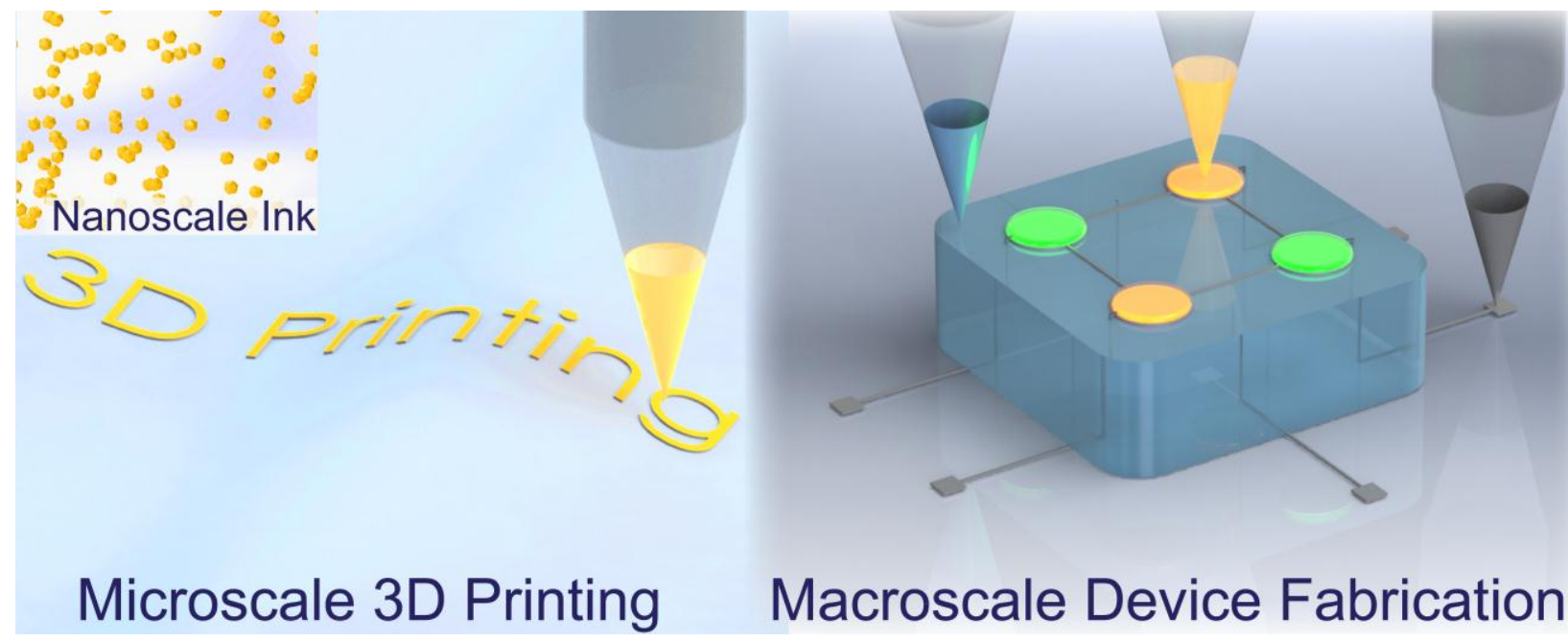

